Library and Archives
Canada

Published Heritage Branch

395 Wellington Street Ottawa ON K1A 0N4 Canada
Bibliotheque et

Archives Canada

Direction du

Patrimoine de l'edition

395, rue Wellington

Ottawa ON K1A 0N4

Canada
Your file Votre reference ISBN: 978-0-494-71556-7 Our file Notre reference ISBN: 978-0-494-71556-7

\section{NOTICE:}

The author has granted a nonexclusive license allowing Library and Archives Canada to reproduce, publish, archive, preserve, conserve, communicate to the public by telecommunication or on the Internet, loan, distribute and sell theses worldwide, for commercial or noncommercial purposes, in microform, paper, electronic and/or any other formats.

The author retains copyright ownership and moral rights in this thesis. Neither the thesis nor substantial extracts from it may be printed or otherwise reproduced without the author's permission.

In compliance with the Canadian Privacy Act some supporting forms may have been removed from this thesis.

While these forms may be included in the document page count, their removal does not represent any loss of content from the thesis.

$$
\text { Canadä }
$$

\section{AVIS:}

L'auteur a accorde une licence non exclusive permettant a la Bibliotheque et Archives Canada de reproduire, publier, archiver, sauvegarder, conserver, transmettre au public par telecommunication ou par l'Internet, preter, distribuer et vendre des theses partout dans le monde, a des fins commerciales ou autres, sur support microforme, papier, electronique et/ou autres formats.

L'auteur conserve la propriete du droit d'auteur et des droits moraux qui protege cette these. $\mathrm{Ni}$ la these ni des extraits substantiels de celle-ci ne doivent etre imprimes ou autrement reproduits sans son autorisation.

Conformement a la loi canadienne sur la protection de la vie privee, quelques formulaires secondaires ont ete enleves de cette these.

Bien que ces formulaires aient inclus dans la pagination, il n'y aura aucun contenu manquant. 


\title{
Fast EMI Analysis of Massively Coupled Interconnects with Long Delay
}

\author{
by \\ Ashok Thookkaparambil Narayanan \\ A thesis submitted to the Faculty of Graduate Studies and Research in partial \\ fulfillment of the requirements for the degree of Master of Applied Sciences \\ Ottawa-Carleton Institute for Electrical and Computer Engineering \\ Department of Electronics \\ Faculty of Engineering \\ Carleton University \\ Ottawa, Ontario, Canada \\ September 2010 \\ (C) Ashok Thookkaparambil Narayanan 2010
}




\begin{abstract}
The recent increase in the operating frequencies and demand for compact as well as low-power electronic devices, has highlighted the previously neglected interconnect and Electromagnetic Interference (EMI) effects in high-speed designs. The success of high-speed designs depend heavily on the accurate and timely estimation of EMI and related interconnect effects like reflection, delay and crosstalk. However, at gigahertz frequencies, conventional EMI and interconnect macromodels become inefficient, especially when applied to massively coupled interconnects with long delay. This inefficiency is mainly because of the large coupled circuit that results from the application of conventional macromodels. Hence, to address this problem, a new algorithm that combines the advantages of recently developed algorithms, namely, the Waveform Relaxation and Transverse Partitioning (an efficient method to simulate coupled interconnects), and the Delay Extraction-based Passive Compact Transmission-line macromodel (an efficient macromodel for long interconnects), is presented. This algorithm achieves a significant reduction in simulation time. Additionally, a parallel EMI analysis algorithm that further decomposes the simulation into time blocks is also presented. This parallel algorithm results in further CPU time savings and is found to scale well with the number of processors.
\end{abstract}


Dedicated to my Mother 


\section{Acknowledgments}

I would like to thank my supervisors, Professor Ram Achar and Professor Michel Nakhla for their guidance and motivation throughout this research. They provided the initial inspiration for this work and gave critical guidance throughout the process. I thank Professor Q. J. Zhang for the fruitful discussions and suggestions.

I am indebted to Douglas Paul for his continuous support and guidance during this research. I am grateful to A. Sridhar and H. Dhindsa for helping me to settle down in Ottawa and for the support during the initial stages of this research. I also thank N. Nakhla for helping me to start this research. I wish to thank my colleagues M. Adel and D. Trifkovic for meticulously proof reading this thesis. I would like to acknowledge and thank my colleagues B. Nouri, Y. Hong, L. Filipovic, A. Charest, J. J. Kang, B. Yogendran, M. Siddique, H. Saini, A. Saini, L. Mutu for the helpful discussions and relaxing chats which made my stay at Carleton a pleasant and enjoyable experience.

I wish to thank Kamalamma and Dr. S. Sourirajan for their affection and guidance. I leave a special note of appreciation for my brother Anil Narayanan for being my extended hands in India performing my responsibilities better than what I would have been able to do. I don't have words to express my thanks for my parents S. Chandrika and T. K. Narayanan for all the support, patience and affection. 


\section{Contents}

1 Introduction 1

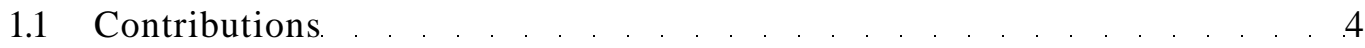

1.2 Organization of the Thesis . . . . . . . . . . . . . . 5

2 Review of Field Coupling Models for Interconnects 6

2.1 Problem Formulation $\ldots \ldots$

2.1.1 Plane wave coupling to MTLs. . . . . . . . . . . . . . 12

2.2 The Solution of Inhomogeneous Telegrapher's Equations. . . . . . 20

2.3 Macromodels for EMI Analysis of MTLs _ _ . . . . . . 22

2.3.1 Lumped Segmentation . . . . . . . . . . . . . . 23

2.3.2 Method of Characteristics . . . . . . . . . . . . 24

2.3.3 Matrix Rational Approximation _. . . . . . . . . . . 27

2.3.4 DEPACT: Delay Extraction-based Compact Macromodeling Al-

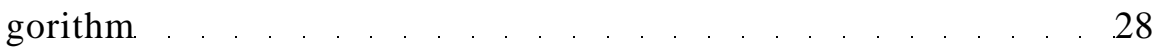


2.4 Summary . . . . . . . . . . . . . . . . . . . . . . . . . . . . . . .

3 Review of Waveform Relaxation $\quad 34$

3.1 The Waveform Relaxation Method . . . . . . . . . 36

3.2 Transverse Partitioning of Transmission Lines . . . . . . 37

3.3 Waveform Relaxation Method for DEPACT macromodel _ . . . 42

3.3.1 Transverse Partitioning of DEPACT Lossy Sections. . . . . 43

3.3.2 Transverse Partitioning of DEPACT Lossless Sections. . . 45

3.4 Summary . . . . . . . . . . . . . . . . . . . . . . . . . 52

4 EMI Analysis of Massively Coupled Interconnects with Long Delay 53

4.1 EMI Macromodel Based on DEPACT (MLF-I) Macromodel . . . 55

4.1.1 Derivation of the Distributed Sources _ _ . . . . . 55

4.1.2 Proposed Decoupled EMI Macromodel (WR-TP + MLF-I) . . 59

4.2 EMI Macromodel Based on DEPACT (MLF-II) Macromodel . . 63

4.2.1 Proposed Decoupled EMI Macromodel (WR-TP + MLF-II) . 63

4.3 Numerical Examples . . . . . . . . . . . . . . . . . . . 65

4.4 Summary . . . . . . . . . . . . . . . . . . . . . . . . 78

5 Parallel EMI Analysis of Massively Coupled Interconnects 79

5.1 Review of EMI Analysis of Interconnects Using Physical Partitioning 81 
5.1.1 Review of Parallel Interconnect Simulation Using Physical and Time Partitioning (PS-PTP) _ . . . . . . 83

5.2 Proposed Parallel EMI Analysis Algorithm. . . . . . . . . . . . . 85

5.3 Numerical Examples. . . . . . . . . . . . . . . . . . . . . . . . . 89

5.4 Summary . . . . . . . . . . . . . . . . . . . . . . . . .

6 Conclusions and Future Work 99

6.1 Future Work . . . . . . . . . . . . . . . . . . . . . . . . . .

$\begin{array}{ll}\text { References } & 101\end{array}$ 


\section{List of Figures}

2.1 n-line MTL structure _. . . . . . . . . . . . . . . 10

2.2 Contour for calculating line voltages . . . . . . . . . . . . . . 11

2.3 (a) Plane wave incidence, (b) The polarization of the incident E-field. 14

2.4 Cross-sectional view of $j^{t h}$ line . . . . . . . . . . . . . . . 16

2.5 Plane wave reflected from a PEC ground plane . . . . . . . . . 18

2.6 RLC section used in lumped segmentation . . . . . . . . . . . . . 24

2.7 Equivalent circuit for MoC macromodel (lossless line) _ . . 25

2.8 Schematic of the DEPACT macromodel (MLF-II.). . . . . . . . 31

2.9 Coupled EMI macromodel based on MLF-II _ . . . . . . . . . . 32

3.1 Transverse partitioning using single-ended sources _ . $\quad 40$

3.2 Transverse partitioning using distributed sources. . . . . . . . . . . 42

3.3 Decoupled DEPACT lossy section _ . . . . . . . . . . . . . 45

3.4 Decoupled DEPACT lossless section . . . . . . . . . . . . . 51 
$4.1 k^{\text {th }}$ DEPACT section . . . . . . . . . . . . . . . 56

4.2 Coupled macromodel based on MLF-I . . . . . . . . . . . . . . . 60

4.3 (a) Proposed macromodel (WR-TP + MLF-I + EMI). (b) $k^{\text {th }}$ DEPACT cell . . . . . . . . . . . . . . . . . . . . . . . .

4.4 (a) Proposed macromodel (WR-TP + MLF-II + EMI). (b) $k^{\text {th }}$ DEPACT cell . . . . . . . . . . . . . . . . . . . . . .

4.5 Example 1: Structure of 2-line MTL _ $\quad 67$

4.6 Example 1: Comparison of transient responses at the far end of line 1. 67

4.7 Example 1: Comparison of transient responses at the near end of line 2. 68

4.8 Example 2: Structure of 3-line MTL . . . . . . . . . . . . . . . . . 69

4.9 Example 2: Comparison of transient responses at the far end of line 1.70

4.10 Example 2: Comparison of transient responses at the near end of line 3. 71

4.11 Example 3: Structure of 10-line MTL … 72

4.12 Example 3: Comparison of transient responses at the near end of line 1. 74

4.13 Example 3: Comparison of transient responses at the near end of line

10.

4.14 Example 3: Simulation time comparison of proposed EMI analysis method (WR-TP + MLF-II + EMI) vs conventional (coupled lumped segmentation) method. 
4.15 Example 3: Simulation time comparison of proposed EMI analysis method (WR-TP + MLF-II + EMI) vs existing (WR-TP + lumped segmentation + EMI) method

5.1 EMI macromodel based on WR-TP and lumped segmentation.

5.2 2-line MTL structure.

5.3 Example 1: Comparison of transient responses at the far end of line 1.91

5.4 Example 1: Comparison of transient responses at the far end of line 1 (With EM field excitation alone).

5.5 Example 1: Comparison of transient responses at the near end of line

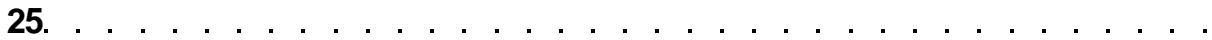

5.6 Example 2: Speed-up vs number of processors graph for the proposed parallel EMI analysis method. 


\section{List of Tables}

4.1 Example 1: Comparison of error norms for MLF-I and MLF-II based models.

4.2 Example 2: Comparison of error norms for MLF-I and MLF-II based models

4.3 Example 3: Simulation time comparison of proposed EMI analysis method (WR-TP + MLF-II + EMI) vs conventional (coupled lumped segmentation) method.

4.4 Example 3: Simulation time comparison of proposed EMI analysis method (WR-TP + MLF-II + EMI) vs existing (WR-TP + lumped segmentation + EMI) method

5.1 Example 2: Simulation time measurements for proposed parallel EMI analysis method. 
5.2 Example 3: Simulation time for proposed parallel EMI analysis method (8 CPUs)

5.3 Example 3: Simulation time for proposed parallel EMI analysis method (4 CPUs). 


\section{List of Symbols}

$£ j \mathrm{~g} \quad$ Electric field vector in time and frequency domain, respectively.

$E Q$ Temporal envelope of the EMI pulse.

$E_{0}$ Fourier transform of the temporal envelope of the EMI pulse.

$A_{x}, A_{y}, A_{z} \quad$ Electric field components of the incident plane wave along the rectangular coordinate axes.

$a_{x}, a_{y}, a_{z} \quad$ Unit vectors along the rectangular coordinate axes.

$K_{X}, K_{y}, K_{Z} \quad$ Wave vectors along the rectangular coordinate axes.

R, L, G, C Per unit length parameters resistance, inductance, conductance, capacitance of the MTL.

T\{.\} Fourier transform operator.

u) Angular frequency of the phasor.

s $\quad$ Laplace variable $s=i u>$.

I Length of the transmission line.

$m \quad$ Number of DEPACT sections used to represent transmission line 
A

B

$\mathrm{Q}(\mathrm{s})$

Qi

r

Y

$\mathrm{T}$

$\mathrm{Y}$

$\operatorname{tt}(\mathrm{i})$

$\mathrm{Up}$

$\mathrm{U}_{\mathrm{n} \times \mathrm{n}}$
Matrix formed by R. and $G$ of transmission line.

Matrix formed by $\mathrm{L}$ and $\mathrm{C}$ of transmission line.

Matrix formed by R, L, G, C parameters of the transmission line.

Product term for modified Lie product formula.

Delay of a transmission line.

Admittance parameter of transmission line.

Transmission parameter of transmission line.

Re-arranged Y parameter of transmission line.

Unit step function.

Selector matrix.

$n \times n$ Identity matrix. 


\title{
Abbreviations
}

\author{
ccNUMA Cache Coherent Non-Uniform Memory Access \\ DEPACT Delay Extraction-Based Passive Compact Transmission-Line Macromodel- \\ ing Algorithm \\ EM Electromagnetic \\ EMC Electromagnetic Compatibility \\ EMI Electromagnetic Interference \\ FFT $\quad$ Fast Fourier Transform \\ ODE Ordinary Differential Equation \\ IFFT Inverse Fast Fourier Transform \\ MoC Method of Characteristics \\ MRA Matrix Rational Approximation \\ MTL Multi-conductor Transmission Line \\ ODE Ordinary Differential Equation \\ PDE Partial Differential Equation
}




$\begin{array}{ll}\text { PEC } & \text { Perfect Electric Conductor } \\ \text { PS-PP } & \text { Parallel Simulation Using Physical Partitioning } \\ \text { PS-PTP } & \text { Parallel Simulation Using Physical and Time Partitioning } \\ \text { p. u. 1. } & \text { Per Unit Length } \\ \text { PWL } & \text { Piece-Wise Linear } \\ \text { TEM } & \text { Transverse Electromagnetic } \\ \text { TP } & \text { Transverse Partitioning } \\ \text { TL } & \text { Transmission Line } \\ \text { UTP } & \text { Unshielded Twisted Pair } \\ \text { VLSI } & \text { Very Large Scale Integration } \\ \text { WR } & \text { Waveform Relaxation } \\ \text { WR - TP } & \text { Waveform Relaxation with Transverse Partitioning }\end{array}$




\section{Chapter 1}

\section{Introduction}

In the recent days, interconnect analysis constitutes a major part of circuit simulations that are performed in high-speed designs. In these designs, the signals attain subnanosecond edge rates with considerable power in the $\mathrm{GHz}$ region of the frequency spectrum. At GHz frequencies, most interconnects are electrically long and behave like transmission lines. Transmission line effects like reflection, delay and crosstalk can severely affect the quality of the signals and in turn the performance of the product.

Signal propagation on long interconnects is also susceptible to electromagnetic interference (EMI) caused by natural as well as man made sources. Long interconnects, in the presence of EMI, act as good antennas by efficiently coupling the radiated noises into the circuit, thereby degrading the signal integrity or even damaging the product. Hence, the capability to simulate long interconnects, usually modeled as 
multi-conductor transmission lines (MTLs) excited by incident electromagnetic fields, is essential for an accurate EMI analysis.

In current designs that have multi-gigabit bus speeds, conventional EMC design rules are not applicable and can even result in product failures. In addition, some of these rules are too restrictive, in that they do not give the designer much scope for improvement. Consequently, EMI analysis using fast solvers is replacing the conventional rule-checking approach for EMC design, as the electronic products are targeting reliability, low cost, higher performance and shorter design cycles.

Fast solvers are built using analytical models [1] derived for specific EMI problems, unlike full-wave EM solvers which directly solve the Maxwell's equations. The analytical model is derived from the Maxwell's equations with simplifying assumptions that are valid for the problem. Using these analytical models, fast solvers allow the designers to simulate multiple scenarios of the EMI problem in a reasonable time. These simulations aid the designers to make critical decisions during the design phase.

The fast EMI analysis algorithms, presented in this thesis, focus on the analysis of massively coupled interconnects excited by radiated fields from far EMI sources. These algorithms use a SPICE-like circuit simulation environment, and hence, allow easy simulation of nonlinear terminations connected to the interconnects. The proposed algorithms are based on field coupling models for transmission lines that formulate the EMI problem in the form of inhomogeneous Telegrapher's equations [2]. 
The simulation of high-speed interconnects and EMI effects described by the Telegrapher's equations is faced with the major challenge of mixed frequency/time domain analysis. This is due to the fact that the solution to the Telegrapher's equations is best described in the frequency domain, however, most practical circuits have nonlinear terminations that can be only be described in the time domain. Therefore, solving such circuits require multiple transformations between the frequency and time domain using FFT/IFFT, in turn making the simulation slow.

To solve this mixed frequency-time problem, several time domain macromodels that approximate the solution of Telegrapher's equations have been proposed [2-5]. However, the effectiveness of these macromodels degrades considerably in the presence of massively coupled interconnects because these macromodels result in large coupled system of equations. Thus making the overall simulation memory inefficient and extremely slow. In order to efficiently simulate massively coupled interconnects, the WR-TP algorithm [6] was recently proposed, which formed the motivation for this work.

Another major challenge faced in EMI simulation is the efficient simulation of long low loss lines. The conventional methods have difficulty handling long transmission lines (resulting in large circuits or higher order approximations), making them computationally inefficient. To overcome this problem, the delay extraction-based passive compact transmission-line macromodel (DEPACT) was proposed [7]. 
A new EMI analysis algorithm is proposed in this thesis, by combining WR-TP algorithm with the DEPACT macromodel. The proposed algorithm can efficiently simulate massively coupled interconnects having long delays.

\subsection{Contributions}

In this thesis, fast algorithms for EMI analysis of massively coupled interconnects having long delays are proposed. The specific contributions made in this thesis are listed below.

1. A new algorithm for EMI analysis is proposed, combining the advantages of waveform relaxation and the delay-extraction-based TL macromodel (DEPACT).

2. An analytic formulation of the equivalent EMI sources and WR sources used in the new algorithm are derived in the time domain, to avoid time/frequency conversions during transient simulation.

3. A new parallel algorithm for EMI analysis that efficiently utilizes multiple processors using the concept of physical and time partitioning [8] [9], is proposed. 


\subsection{Organization of the Thesis}

This thesis is organized as follows. Chapter 2 provides a review of field coupling analysis of interconnects using existing macromodels. Chapter 3 provides a review of WR-TP method for the simulation of interconnects. Chapter 4 presents the details of the proposed algorithm for fast EMI analysis of interconnects and numerical examples.

Chapter 5 presents the details of the proposed parallel algorithm for EMI analysis and numerical examples showing the scalability of the algorithm on multiple cores. Finally, in Chapter 6, conclusions are made, and few ideas for future research are presented. 


\section{Chapter 2}

\section{Review of Field Coupling Models}

\section{for Interconnects}

The most accurate method for analyzing an interconnect excited by an electromagnetic field is to use a full-wave solver [10], which solves Maxwell's equations on a $3 \mathrm{D}$ model of the interconnect structure and the surrounding medium. However, this method is computationally expensive and requires a significant modeling time. Also, it is very difficult to interface these full-wave models with SPICE-like nonlinear circuit simulators due to the mixed frequency/time problem.

A significant simplification can be achieved by modeling the interconnect behavior using transmission lines that are based on TEM analysis, where the electric and magnetic fields are assumed to be limited to the transverse plane. However, TEM 
approximation is only valid for the case of a uniform transmission line made of perfect conductors in a homogeneous medium. Instead, a more practical approach is to use the Telegrapher's equations [11], which is based on a quasi-TEM assumption. In the case of a quasi-TEM assumption, a small component of the fields in the longitudinal direction is also considered. These longitudinal fields can account for the small losses in the conductors and inhomogeneities in the medium observed in practical interconnects.

Field coupling models derived using a quasi-TEM assumption can accurately predict the load response to EMI, in most practical cases. It is worth noting that interconnects excited by a field can have transmission line mode (differential mode) as well as antenna mode (common mode) currents propagating. Even though quasiTEM models only give the solution of transmission line mode currents, these models also yield good approximations for the load response due to the following reasons [1]. The antenna mode currents typically reduce to zero at the loads, therefore the accuracy of the load response is not affected. Moreover, in the case of transmission lines that are referenced to a ground plane, the effects of antenna mode currents can be reasonably neglected. Hence, a quasi-TEM based method for field coupling analysis is used in this thesis.

This chapter is organized as follows. In section 1, the formulation of the EMI analysis problem using an existing field coupling model is reviewed. In section 2, 
the solution of the resulting inhomogeneous Telegrapher's equations is reviewed. In section 3, various macromodeling algorithms that can be used to model the solution of inhomogeneous Telegrapher's equations are reviewed.

\subsection{Problem Formulation}

In the EMI problem considered here, we are interested to know the response at the terminations of a MTL excited by an EM field. Analysis of field coupling to MTLs have been widely studied in the literature [2,12-17].

These existing field coupling models represent the EM excitation using distributed voltage and current sources connected along the length of the MTL. The distributed excitation of a MTL can be represented using inhomogeneous Telegrapher's equations. Two popular formulations for these equations are available in the literature [1], which differ in the way the forcing function (inhomogeneous part) is calculated.

1. Taylor's method: Distributed voltage and current sources are used to represent the magnetic flux linking the conductors and electric flux terminating on the conductors, respectively.

2. Agrawal's method: Distributed voltage sources alone are used to represent the electric field along the conductors. These electric fields are calculated by considering field coupling as a EM scattering problem. 
These two methods are equivalent [1], however, Agrawal's method was chosen here, as it leads to a simpler formula for the distributed sources.

Consider an n-line MTL excited by an electromagnetic field, as shown in Figure 2.1. The geometry of the MTL satisfies the conditions for the quasi-TEM assumptions to be valid, namely, $d<\mathrm{C} I$, and $w<\mathrm{C} d$; where $d$, I and $w$ are the separation, length and width of the lines, respectively.

In Agrawal's method, the total field around the conductors is expressed as the sum of incident field and scattered field. The incident field is the field that is present when the conductors are removed. The scattered field is the field produced by the currents and charges induced on the conductors. Then, the transverse and longitudinal electric field around the conductors can be written as

$$
\begin{aligned}
& \mathrm{E}_{\mathrm{t}}=\mathrm{EJ}^{\prime c}+\mathrm{Ef} \\
& \mathrm{E}_{2}=\mathrm{Ef}^{\mathrm{c}}+\mathrm{Ef}^{*}
\end{aligned}
$$

where the subscripts $t$ and $z$ denote transverse and longitudinal fields, respectively. Now, the voltage on $j^{t h}$ line due to scattered field can be defined as [2]

$$
V f^{c t}(z, t)=-f \quad E f \cdot 51
$$




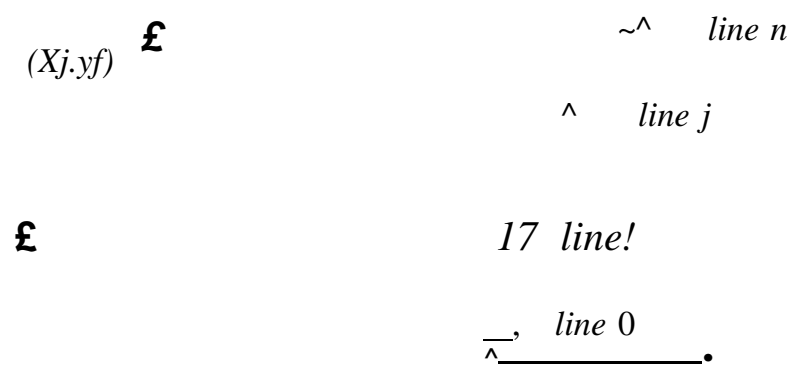

Figure 2.1: n-line MTL structure.

where the line integral in (2.2) is defined in the contour shown in Figure 2.2.

Also, the total voltage of $j^{t h}$ line is related to the scattered voltage as

$$
V_{3}(z, t)=V_{3}^{s a}\{z, t)+V^{\mathrm{TM}}(z, t)
$$

where

$$
\boldsymbol{v}^{\mathrm{TM}}(\mathrm{z}, \boldsymbol{t})=-\underset{J a}{\boldsymbol{r}} \boldsymbol{E} ? \cdot 51
$$

It is to be noted that the above relation (2.3) will be used later to obtain the total voltages from the scattered voltage and would be represented as terminal sources in the macromodel. 
(xj.yj-) $\quad$ line $j$

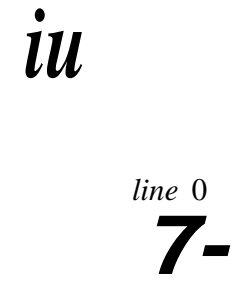

Figure 2.2: Contour for calculating line voltages.

Next, the inhomogeneous Telegrapher's equations representing the field coupling to MTLs can be written as [2]

$$
\begin{aligned}
-\mathrm{V}^{\mathrm{sct}}(\mathrm{z}, t)+K L(z, t)+\mathrm{L}-\mathrm{I}(\mathrm{z}, t) & =V_{F}(z, t) \\
\mathrm{J} \wedge \mathrm{ICM})+G V^{s c t}(z, t)+C^{\wedge} V^{s c t}(z, t) & =0
\end{aligned}
$$

where $\mathrm{V}^{\wedge}$ is the forcing function defined as

$$
V_{F}(z, t)=\& \%(z, t)-E^{\mathrm{TM}}\{z, t)
$$


and $I(z, t)$ is the row vector of line currents as a function of position $z$ and time $t$; $Y^{s c t}$ is the row vector of voltages on the lines due to the scattered field; $\mathrm{R} \mathrm{G} \mathrm{R}^{\text {"Xn }}$, $\mathrm{L} G \mathrm{~K}^{\mathrm{nxn}}, \mathrm{C} \mathrm{G}^{\mathrm{n}} \mathrm{M}^{\mathrm{x}}$ and $\mathrm{G} \mathrm{G} \mathrm{R}^{\prime \mathrm{x}}$ " are the per-unit-length (p.u.l) resistance, inductance, capacitance and admittance parameter matrices, respectively. These p.u.l parameters can be extracted from the cross sectional dimensions of the uniform structure, using a field solver such as [18]. $\mathrm{E}^{* \mathrm{n}} ?(; \mathrm{z}, \mathrm{t})$ is the longitudinal electric field along the position of $j^{t h}$ line, with all the lines removed. $\mathrm{E}^{* \mathrm{TM}}(z, t)$ is the electric field along the reference conductor.

Using a plane wave assumption for the incident fields, the expression for the sources in (2.2) and (2.6) can be simplified. The details of plane wave coupling to MTL is given in the next section.

\subsubsection{Plane wave coupling to MTLs}

Radiated fields of EMI sources can be approximated using a uniform plane wave in the far-field regions of the source. Consider a plane wave incident at the origin of the coordinate system, at an angle $4>$ from the $y$ axis and 8 from the $x$ axis, as shown in Figure 2.3a. The polarization of the electric field vector is shown in Figure 2.3b. The plane wave can be represented using phasor notation, in the Cartesian coordinates as

$$
1^{\mathrm{inc}}(\mathrm{s}, y, z, s)=E_{0}\left(A_{x} a_{x}+A_{y}^{\wedge}+A_{z} S i_{x}\right) e^{-\wedge} \wedge^{x+\wedge} \wedge K_{\prime \wedge}
$$


where

$E_{0}=\wedge\{E Q\}$ is the Fourier transform of the time-domain envelope of the EMI pulse;

$A_{x}, A_{y}, A_{z}$ are the direction cosines;

$\mathrm{a}_{\mathrm{x}}$, ay, $a_{z}$ are the unit vectors;

$K_{X}, K_{y}, K_{Z}$ are the wave vectors;

$s=i c u$ where $U J$ is the angular frequency of the phasor and $i$ is the imaginary number $y f-1$.

The direction cosines and wave vectors can be found from the geometry of the wave incidence as [2]

$$
\begin{aligned}
& A_{x} \sim \sin B_{E} \sin 6 \\
& A y=-\sin B E \cos 6 \cos \langle f\rangle-\cos B E \sin 0 \\
& \left.\left.A_{z}=-\sin B E \cos 6 \sin 4\right\rangle+\cos B E \operatorname{Cos} 4\right\rangle \\
& A^{2}{ }_{x}+A^{2}{ }_{y}+A l=l
\end{aligned}
$$




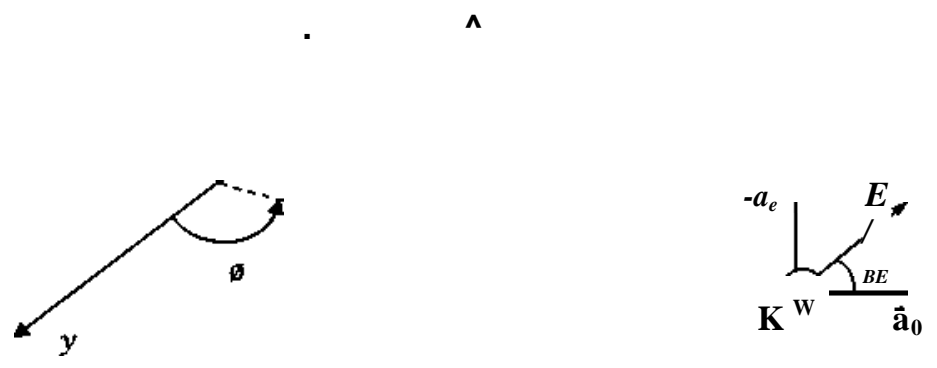

(a)

Figure 2.3: (a) Plane wave incidence, (b) The polarization of the incident E-field.

$$
\begin{aligned}
& \text { AC 7. }-\quad \begin{array}{c}
\cos 9 \\
\mathrm{c}
\end{array} \\
& \sin 6 \cos < \\
& \text { ky - c } \\
& \boldsymbol{K} \boldsymbol{r}=\sim \sin 9 \sin c
\end{aligned}
$$

Next, we consider two special cases of plane wave coupling to MTL: (1) when a conductor at the origin is used as a ground return, (2) when a perfect electrically conducting (PEC) ground plane is used as a ground return. 


\section{Case 1: Ground Conductor at the Origin}

Substituting the field components (2.7) in (2.6), and placing the reference conductor at the origin, an expression for the forcing function sources can be written in the frequency domain as [19]

$$
V_{F t j}(s, z)=E_{0}\left(A_{z} e^{S K_{* z}}\left(e-e^{s}\left({ }^{K x}{ }^{+}+K_{y}{ }^{y} i^{l}-1\right)\right)\right.
$$

where the subscript $j$ denotes the sources for $j^{t h}$ line, $X j, y j$ is the location of the $j^{t h}$ line. Using the first order Taylor series approximation for the exponential term, we obtain a simplified formula [19] for the forcing function

$$
V_{F J}(s, z)=s e^{S K_{* z}} E_{Q} A_{z}\left(K_{x X j}+K_{y y j}\right)
$$

Next, to simplify the formula for the terminal sources (in (2.3)-(2.4)), we substitute (2.7) in (2.4), and write the line integrals in terms of cross-sectional coordinates

$(x j, y j)$ of $j^{t h}$ line. Let $\left(x_{a}, y_{a}\right)$ be a point on the contour (line from the origin to $(x j, y j)$ in Figure 2.4). Then, 


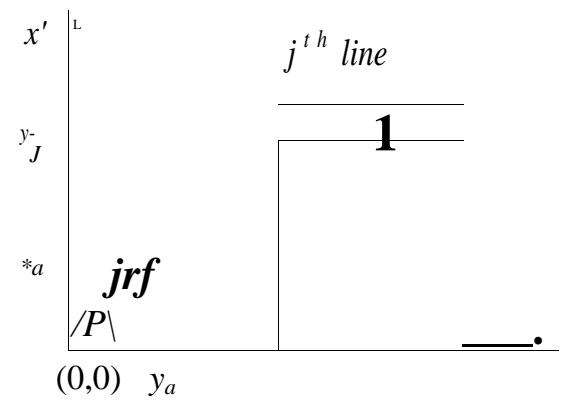

Figure 2.4: Cross-sectional view of $j$ line

$$
\begin{aligned}
& P=V^{X} a^{2}+V a^{2} \\
& d j={ }^{\wedge} X j^{2}+v_{j}^{2} \\
& X_{a}=P- \\
& V a=P_{d j}
\end{aligned}
$$

Substituting (2.7) and (2.12) in (2.4)

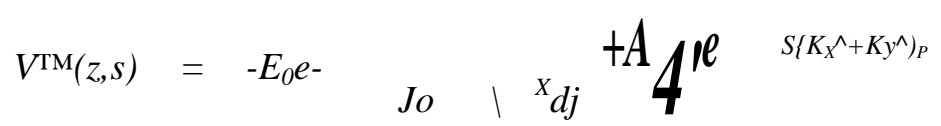




$$
V^{\mathrm{TM}}(z, s)=-E_{0} e^{S K} *^{Z} \wedge^{x X j}+A y V j \quad\left[1-e^{\left.-*^{K} *{ }^{x} i^{+K} \gg r i\right]}\right.
$$

Approximating the exponential using first order approximation [2], we obtain a simplified formula for the terminal sources in the frequency domain [19]

$$
V \&(z, s)=-E_{0} e^{\sim^{\wedge} \wedge}\left\{A_{x} x_{3}+A_{y} y j\right)
$$

\section{Case 2: PEC Ground Plane}

Considering the case of lines referenced to a perfectly electrically conducting (PEC) ground plane, a reflected field is also present around the conductor, as shown in Figure 2.5. Using the PEC boundary condition, the reflected fields can be written as [2]

$$
E^{r e f}=E_{0}\left(A_{x} a_{x}-A_{y} a_{y}-A_{z} a_{z}\right) e^{S K \iota x} e_{-}^{S K} y^{y} e^{\sim^{S} z}
$$

The total field around the conductors, when conductors are removed can be written

as

$$
E^{t o t}=E^{i n c}+E^{r e f}
$$

Using the expression for incident and reflected waves (2.7 and 2.16) in (2.17), the resulting electric field in the $z$ direction along the $j^{t h}$ line can be written for the case 


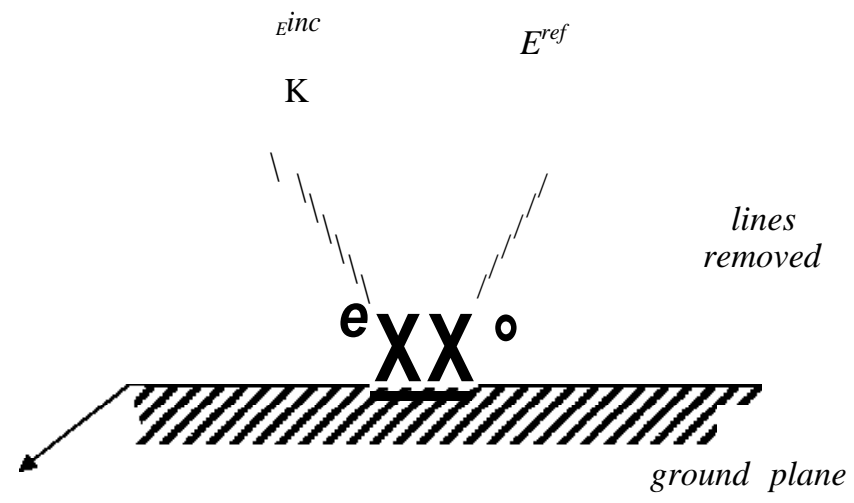

Figure 2.5: Plane wave reflected from a PEC ground plane.

of an infinite PEC ground plane as [2]

$$
E \%(z, s)=-2 i E_{Q} A_{z} \operatorname{sm}\left\{u K_{x} x\right) e^{-S K} y^{y} e-
$$

Using (2.18), the forcing function in (2.6) for the case of a PEC ground plane can be written as

$$
\left.V F(Z, S)=E l^{\circ} t(z, s)-E i \% z, s\right)
$$

where $E_{f} f l(z, s)=0$. 
Since the cross-sectional coordinates $X j$ and $y j$ are electrically short, the quantities $U) K_{x} X j-{ }_{\wedge}^{7 \mathrm{rXl}}$ and $t J K_{y} y j=-^{\wedge}$ are small. Then, $\operatorname{Vpj}$ in (2.19) can be approximated as [2]

$$
V_{F J}(z, s)=-2 s E o A_{z} n_{x X j} e^{S} S{ }_{z z} Z
$$

Hence, the forcing function for the inhomogeneous Telegrapher's equation in (2.5) can be written as

$$
\mathrm{F}(\mathrm{a})={ }_{0}^{V F}=e^{-S K_{* z}} r(s)
$$

where

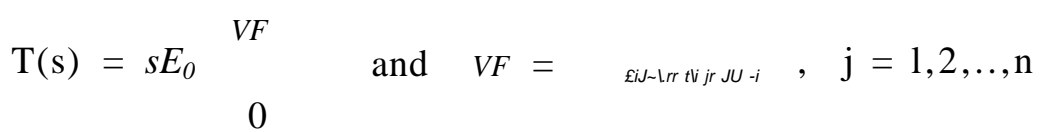

Similarly, the terminal sources given by the integral in (2.4) can be approximated for the case of a PEC ground plane as

$$
\begin{gathered}
V_{t} f(z, s)=-2 E_{0} A_{x X j} e- \\
V_{t}^{i n c}(z, s)=-2 E_{0} e \sim^{s \wedge}
\end{gathered}
$$


where

$$
\mathrm{V}_{7} \quad-Z J i_{x} X j \quad, \quad \mathrm{j}=1,2, \ldots, \mathrm{n}
$$

So far, we have formulated the inhomogeneous Telegrapher's equations and simplified formula for its forcing function representing the field excitation.Next, we consider the solution of these equations.

\subsection{The Solution of Inhomogeneous Telegrapher's Equations}

A time-domain solution of the Telegrapher's equation is desirable for incorporating them in a nonlinear simulator, however, for the case of lossy MTL, there does not exist a closed-form solution in the time domain. Hence, first we solve these equations in the frequency domain and later we approximate these solutions in the time domain.

The Telegrapher's equations (2.5)-(2.6), representing field coupling to MTLs, can be expressed as a set of coupled ordinary differential equations (ODE) in the frequency domain as

$$
\begin{array}{lll}
V^{s c t}(z: s) & - & V^{s c t}(z, s) \\
I(z, s) & \mathrm{I}(\mathrm{z}, \mathrm{s})
\end{array}+\mathrm{F}(\mathrm{z}, \mathrm{s})
$$




$$
\begin{array}{ccc}
\mathrm{Q}(\mathrm{s}) & 0 & -\mathrm{R}-\mathrm{sL} \\
& -\mathrm{G}-\mathrm{s} \mathrm{C} & 0 \\
F(z, s)=\begin{array}{c}
V f(z, s) \\
0
\end{array} & ; V^{\wedge}(z, s) & \%(\mathrm{z}, \mathrm{s})-\mathrm{E}^{\wedge} \mathrm{c}(\mathrm{z}, \mathrm{s})
\end{array}
$$

The solution of the ODEs in (2.26) can be written as

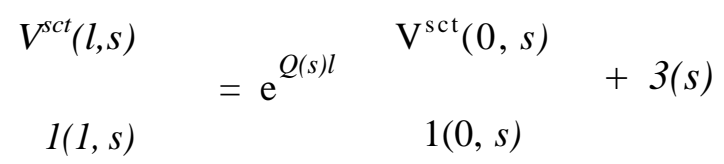

where

$$
\mathrm{J}(\mathrm{s})={ }_{J_{o}} f \quad e^{\left.Q_{\wedge} l_{-} z\right)} F(z, s) d z
$$

Noting that the line voltages due to the scattered field $V ?^{c t}$ are related to the total voltages of the lines as (2.4) (a simplified expression for the terminal sources have been derived in (2.23)), the solution (2.29) can be expressed in terms of total voltages as

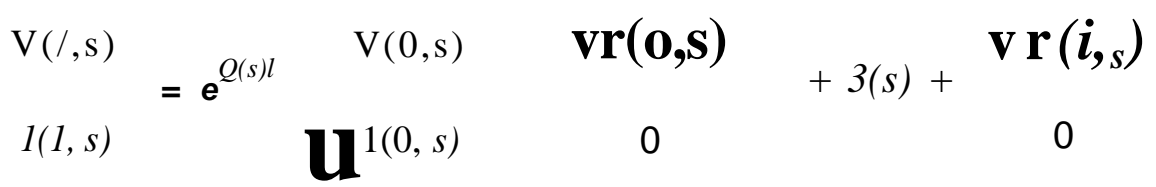

where $\mathrm{Vj}^{\mathrm{nc}}(0, s), V_{t}^{l n c}(l, s)$ represent the sources at the near and far terminals of the 
lines, respectively, and $\mathrm{J}(\mathrm{s})$ represent the distributed EMI sources.

The matrix exponential in (2.31) can be easily evaluated in the frequency domain, however, it does not have an equivalent closed-form expression in the time domain. Hence, interfacing this solution with a nonlinear simulator like SPICE becomes difficult, and is referred to as mixed frequency/time problem [11]. To solve this problem, several time-domain macromodels for transmission lines have been proposed in the literature [7,20-22]. Also, several methods for EMI analysis of transmission lines have been proposed $[23,24]$. Some of these macromodels that are relevant to this thesis are reviewed in the following section.

\subsection{Macromodels for EMI Analysis of MTLs}

Macromodels such as lumped segmentation, method of characteristics (MoC), matrix rational approximation (MRA) and delay extraction-based passive compact transmissionline macromodel (DEPACT) have been proposed to overcome the mixed frequency/time problem. Macromodels approximate the matrix exponential (2.29) in the time domain using equivalent circuits, and the EM excitation using distributed sources. A brief review of these macromodels is given in this section. 


\subsubsection{Lumped Segmentation}

The lumped segmentation model [20] represents a given transmission line using a cascade of sections containing RLC lumped elements connected in a $T$, II or $\mathrm{T}$ arrangement. Each lumped section has a length $A z$ that is electrically small at the frequency of interest. The EM excitation is represented using voltage and current sources connected to each lumped section. The lumped segment model can be described using the Telegrapher's equations discretized in space as

$$
\begin{aligned}
& \mathrm{v}_{\mathrm{fc}}+\mathrm{i}(\mathrm{t})-\mathrm{v}_{\mathrm{fc}}(\mathrm{t})=A z \mathrm{Ai}_{\mathrm{fc}}(\mathrm{i})+\mathrm{AzL}-\mathrm{i}_{\mathrm{fc}}(\mathrm{t})+v_{E E}\left(z_{k}, t\right) \\
& \text { at }
\end{aligned}
$$

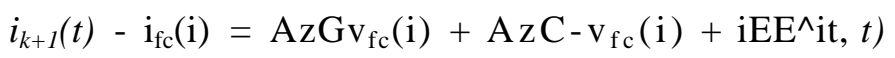

where $A ; G\{1, \ldots, m\}$ is the section index, $m$ is the number of sections, $\mathrm{Az}=\mathrm{l} / \mathrm{m}$, and $\operatorname{VEE}(z k, t)$ and $i E E\{z k, t)$ are the equivalent EMI sources for the $k^{\text {th }}$ section. A schematic of a lumped section for the case of a single line is shown in Figure 2.6.

Note that, at high frequencies, the number of sections required for a lumped segmentation model can be very high as indicated by the formula [11]

$$
m=1(\mathrm{M}
$$

where $T j=y L j j C j j$ is the p.u.l delay of the line and $t_{r}$ is the rise time of the prop- 


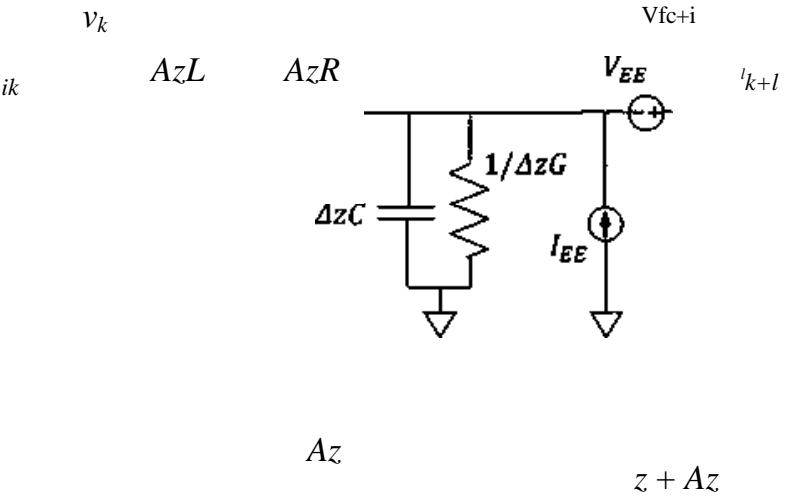

Figure 2.6: RLC section used in lumped segmentation

agating signal. The large number of sections required by the lumped segmentation model can result in excessive simulation time.

\subsubsection{Method of Characteristics}

The method of characteristics (MoC), originally proposed for lossless lines [21], provides an analytical model for transient simulation of transmission lines. The circuit equivalent of this model can be represented using time-delayed sources and the characteristic impedance $Z_{0}$ as shown in Figure 2.7. The delayed sources are defined in (2.34) 
$V l$

$w_{2}$

Figure 2.7: Equivalent circuit for MoC macromodel (lossless line).

$$
\begin{aligned}
& \left.w^{\wedge} t+r\right)=2 v_{2}(t)-w_{2}(t) \\
& w_{2}(t+r)=2 v_{V l}(t)-w_{l}(t)
\end{aligned}
$$

The MoC was extended to include field coupling to the lines [3], by adding sources representing the field coupling to the matched scattering parameter formulation of transmission lines. The scattering parameter formulation for a single line with an incident field can be written as [3,25]

$\begin{array}{ccccc} & S n & S \backslash_{2} & & B f i \\ B_{2} & S_{2} i & S_{22} & A_{2} & B_{f 2}\end{array}$


where $A i, A 2, B i, B_{2}$ are current waves. $B f i, B f_{2}$ are the equivalent sources due to the incident field, which are given by

$$
\begin{gathered}
B f i=-\sim^{\mathbf{i}} / e^{r i}{ }^{-\wedge^{z}}\left(Y_{c}(s) V_{d}-I d\right) d z \\
\left.B f_{2}=-\backslash f e^{-\wedge^{l}-\uparrow} Y_{c}(s) V_{d}+I d\right) d z
\end{gathered}
$$

where $Y_{c}(s)$ is the characteristic admittance of the line, $V \&$ and $I d$ are the distributed sources due to incident fields, and $7(\mathrm{~s})$ is the propagation constant given by

$$
{ }_{7}(\mathrm{~s})=y /(R+s L)\{G+s C)
$$

To implement this formulation in a nonlinear simulator, (2.35) has to be transformed into a circuit represenation in the time domain. However, transforming the transcendental functions of s: $B f i, B f i, \quad \mathrm{e} \sim^{7} \wedge$ requires numerical Laplace inversion or rational function approximation, making it computationally expensive. Moreover, MoC does not guarantee passivity when used for lossy MTLs, hence, this model does not guarantee the stability of transient simulation of the overall circuit. 


\subsubsection{Matrix Rational Approximation}

The matrix rational approximation (MRA) was proposed [22] for macromodeling lossy lines while ensuring passivity. The MRA macromodel approximates the transmission line stamp (2.29) using the Pade approximation [11] as given in (2.39). It is to be noted that the coefficients of the Pade approximation can be pre-calculated using closed-form formulas in the case of exponential function.

$$
=(\mathrm{A}+\mathrm{sB}) \mathrm{i}-\begin{array}{cc}
\mathrm{Tn} & \mathrm{T} 12 \\
\mathrm{~T} 21 & \mathrm{~T}_{2} 2
\end{array} \quad[\mathrm{Xiv}(\mathrm{Z})]-{ }^{1} \wedge_{\mathrm{J}} \mathrm{V}(\mathrm{Z})
$$

where $X N(Z), \quad$ fijv $(Z)$ are polynomial matrices given by

$$
X N(\boldsymbol{Z})=\boldsymbol{J}_{i=0}^{N} \mathbf{\Lambda}^{\prime} \quad \boldsymbol{n}_{N}(z)=\underset{\mathrm{i}=0}{N}, \boldsymbol{Q} \boldsymbol{V}
$$

and $Q, \&$ are the pre-calculated coefficients. A field coupling model based on MRA was proposed in [4]. In this method, the equivalent EMI sources represented by $J(s)$ in (2.30) are approximated as rational functions. Then the solution of the Telegrapher's equation can be written in the form of admittance parameters as

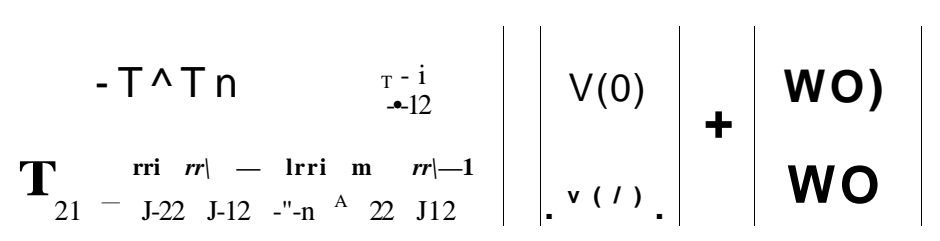




$$
\begin{array}{llll}
\operatorname{Isc}(\mathrm{O}) & -\mathrm{x}_{12}^{-\mathrm{i}} & 0 & \mathrm{~J}(\mathrm{~s}) \\
\operatorname{Isc}(l) & -\mathrm{T}_{2} 2 \mathrm{~T}_{12} & \mathrm{IL}, &
\end{array}
$$

where $\operatorname{Isc}(\mathrm{O})$ and $\operatorname{lsc}\left(0^{\text {are }}\right.$ equivalent EMI sources at the terminals of the line. Since all the terms in (2.42) are expressed as rational functions, it can be easily synthesized in the time domain and simulated using a nonlinear simulator. However, in the case of long delay lines with low loss, the rational expressions typically require higher orders for good approximations, thus limiting the application of this method to relatively short lines.

\subsubsection{DEPACT: Delay Extraction-based Compact Macromod- eling Algorithm}

The DEPACT macromodel [7] combines the advantages of both MoC and MRA macromodels, i.e. it is compact like $\mathrm{MoC}$ and ensures the passivity of the model like MRA. It is based on the modified Lie product formula (MLF) [26]. In the DEPACT macromodel, the matrix exponential of a transmission line (2.31) is approximated using a product of exponential terms that can be easily represented in the time domain. The the Modified Lie product formula-I (MLF-I) approximation of matrix 
exponential can be written as

$$
{ }^{3(\mathrm{~A}+\mathrm{sB})} \boldsymbol{1}^{*} \prod_{i=1} \Lambda
$$

where

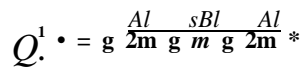

$$
\begin{aligned}
& A=\begin{array}{cc}
0 & -\mathrm{R} \\
\mathrm{G} & 0
\end{array}|; \mathrm{B}=| \begin{array}{cc}
0 & -\mathrm{L} \\
-\mathrm{C} & 0
\end{array}
\end{aligned}
$$

Here, $m$ is the order of approximation for the DEPACT macromodel.

In the case of long low-loss lines, it is more accurate to use Modified Lie product formula-II (MLF-II) approximation [7]

$$
{ }_{0(A+s B) l} \Lambda
$$

where

$$
Q
$$

It is to be noted that the difference between MLF-I and MLF-II is that the former splits the lossy term $e^{A l}$ into two terms, whereas the later splits the lossless term $e^{s B l}$ into two terms. DEPACT macromodel (MLF-II) can be realized using a cascade of $m$ DEPACT sections, each of which consists of one lossy resistive network embedded 
between two lossless delay elements as shown in Figure 2.8.

Later, in [5], EMI effects were incorporated into the DEPACT macromodel and closed-form formulas have been derived for the equivalent EMI sources. It was shown that the EMI effects represented by the convolution integral in (2.30) can be represented as a set of distributed sources $v j$, id and terminal sources $v_{a}, i_{a}, v_{c}, i_{c}$ as shown in Figure (2.9). These sources are defined for the $k^{\text {th }}$ DEPACT section as [5]

$$
\begin{gathered}
\mathrm{v}^{*(\mathrm{t})} \quad=E_{0}\left(t-K_{z} k j-\right) y \\
=-E_{0}\left(t-K_{z}(k+l) J-\right) c p \\
\left.\mathrm{v} \$(\mathrm{t}) \quad K_{z}(k+-)-\right) \quad \mathrm{U}, \\
E_{0}(t-m
\end{gathered}
$$$$
¥
$$

where

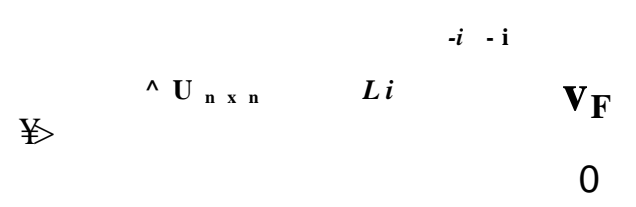




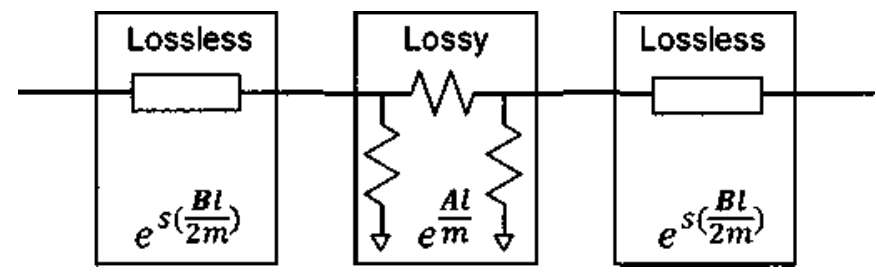

Figure 2.8: Schematic of the DEPACT macromodel (MLF-II.)

As we saw earlier in this chapter, two additional terminal voltage sources $v_{t}^{l n c}(0, t)$ and $v l^{n c}(l, t)$ are required to get the total voltage from the scattered voltage.

$$
\begin{aligned}
& v l^{n c}(0, t)=E_{0}(t) V_{T} \\
& v i^{n c}(d, t)=E_{0}\left(t-n_{z} d\right) V_{T}
\end{aligned}
$$




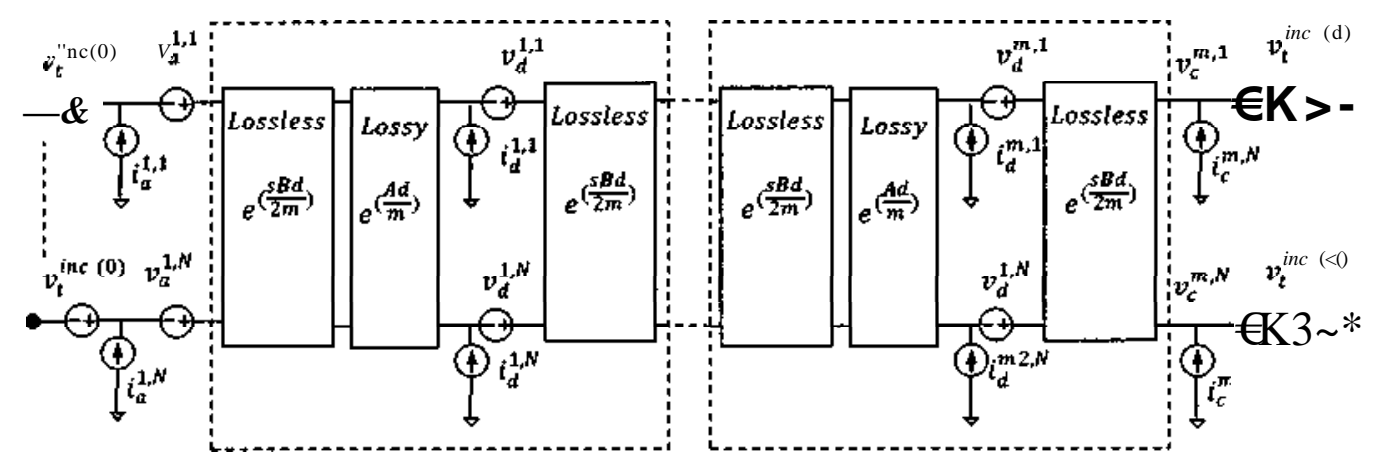

Figure 2.9: Coupled EMI macromodel based on MLF-II 


\subsection{Summary}

EMI analysis of interconnects plays an important role in the recent high-speed designs. In this chapter, we reviewed the existing EMI analysis methods that use the inhomogeneous Telegrapher's equations to model field coupling to MTLs. Next, the solution of Telegraphers's equations in frequency domain and the existing timedomain macromodels for field coupling to MTLs were reviewed. More importantly, a DEPACT based EMI macromodel was reviewed that would be used in the later chapters to derive the proposed EMI analysis macromodel for long interconnects. In the next chapter, a review of fast solution techniques for massively coupled interconnects is given. 


\section{Chapter 3}

\section{Review of Waveform Relaxation}

The waveform relaxation method [27] was first proposed in 1982, for transient analysis of large scale integrated circuits, as an attempt to provide a faster alternative to direct solvers. The key idea in WR is to decompose the circuit into smaller subcircuits that can be solved independently and to arrive at the overall simulation result using an iterative process. In this chapter, first a brief review of the WR method for simulation of general circuits is given. Then, the WR method is reviewed with a specific focus on the simulation of interconnects, namely the waveform relaxation and transverse partitioning (WR-TP) is discussed. Finally, a delay-extraction based WR method suitable for long delay interconnects is reviewed.

Transient simulation of distributed high-speed interconnects and EMI effects using conventional SPICE-like nonlinear simulators typically involve the following steps: 
1. The Telegrapher's equations are discretized using appropriate macromodels to obtain a time-domain circuit representation of the distributed transmission line.

2. Equivalent EMI sources obtained from the field coupling models are calculated.

3. Stable integration methods such as the Backward Euler method and Trapezoidal rule are applied to the circuit equations, which are in the form of ODEs. These integration methods convert the ODEs into a set of algebraic equations. In the case of nonlinear terminations, a set of non-linear algebraic equations are obtained.

4. Nonlinear algebraic equations are solved using Newton iterations. Each iteration requires one LU decomposition of the circuit MNA and a forwardbackward substitution.

For the case of massively coupled interconnects, the first step results in a large coupled circuit, whose size grows proportional to $\mathrm{n}^{2}$, where $n$ is the number of lines. In the subsequent steps, this circuit is solved using conventional simulators that use direct LU solvers. The CPU expense for LU decomposition of a sparse linear system is proportional to $N^{a}$, where $N$ is the size of the MNA and $1.5<a<2$. Hence, the CPU expense of the overall interconnect simulation of $n$ coupled lines is proportional to $n^{13}$, where $3<(3<4$ [6]. This relation shows that for a large number of coupled 
lines, the simulation time can become prohibitively high. Moreover, the number of lines that can be simulated efficiently is limited by the memory capacity of the computer that is used to store the MNA information and the EMI sources.

These limitations can be overcome using decomposition methods that partition the simulation problem into smaller manageable pieces, which can be simulated more or less independently. Such a method based on waveform relaxation, called waveform relaxation and transverse partitioning (WR-TP) was recently proposed [6]. Before we go into the details of WR-TP, the WR method is briefly reviewed in the next section.

\subsection{The Waveform Relaxation Method}

Transient simulation using the waveform relaxation method involves two key steps [27]: the partitioning process and the relaxation process. The partitioning process decomposes the system of nonlinear ODEs representing the circuit, into subsystems of nonlinear ODEs. Each subsystem of ODEs physically represents a subcircuit, and can be simulated independently using conventional methods. Each subcircuit is connected to WR sources that represent the coupling between the subcircuits. The voltage and current variables of each subcircuit are referred to as its internal variables, and all other variables external to the subcircuit are called the external variables with respect to that subcircuit.

The relaxation process is an iterative process. The relaxation process is started 
with an initial guess of the WR sources. Using these WR sources, the individual subcircuits are simulated for the whole time interval of interest, so as to obtain the updated values of the internal variables. The WR sources are updated after every iteration using the current values of all the variables. This process is iterated until all the variables converge within an acceptable tolerance. Next, we review the WR-TP method suitable for interconnect networks.

\subsection{Transverse Partitioning of Transmission Lines}

In the transverse partitioning (TP) approach, a system of coupled transmission lines is subdivided into single lines. Coupling between the lines is represented using waveform relaxation sources. The mathematical formulation of the relaxation sources and the decoupled circuit equations derived in [6] are reviewed here.

Consider a MTL having $n$ conductors, represented using the homogeneous Telegrapher's equations in the time domain

$$
\begin{aligned}
& -v(z, t)=-R i(z, t)-L-i(z, t) \\
& -i(z, t)=-G v(z, t)-C-v(z, t)
\end{aligned}
$$

where R, L, G, C are the p.u.l parameter matrices of the MTL, $v(z, t)$ and $i(z, t)$ are the vectors of voltage and current at the position $z$ of the MTL and the time $t$, 
respectively. From (3.1), the equations for the $j$ line can be written as

$$
\begin{aligned}
& \stackrel{d}{-V j\{z, t)=-\operatorname{Rjjij}\{z, t)} \underset{\left.-L j j-i^{\wedge} z, t\right)+e j(z, t)}{d} \\
& \begin{array}{lll}
d & \sim & -
\end{array} \\
& \left.f c i j(z>t)=-G j j V j i z, t)-C j j-v^{\wedge} z, t\right)+{ }_{q j}(z, t)
\end{aligned}
$$

$$
\begin{gathered}
e,(z, t)=\underset{\mathbf{P}=\mathbf{I}}{\wedge}\left(R-j p i p+L_{j p}-i p\right) \\
\left.\left.q j(z, t)=\stackrel{\substack{n \\
\mathbf{P}=\mathbf{I}}}{(\text { Gjpivj }-V p)+C j p-\wedge} \stackrel{r}{-} i v j-v_{p}\right)\right)
\end{gathered}
$$

where $G j j=\mathrm{X}) \mathrm{p}=\mathrm{i} G j p^{\text {an }} \wedge C j j=Y^{\wedge}=i C j p^{\text {are }}$ the self admittance and capacitance of the line, respectively. The terms $e j(z, t)$ and $q j(z, t)$ represent the coupling effects due to the neighboring lines, on the $j^{\text {th }}$ line. The equation for the $j^{t h}$ line (3.3) can be simplified in the frequency domain into an inhomogeneous ODE of the form

$$
\left.{ }^{\wedge} \mathrm{X},(\mathrm{z}, s)=F j X j i z, s\right)+¥,-(*, s)
$$


where

$$
\begin{aligned}
& \mathrm{X}_{\mathrm{J}}-(\mathrm{z}, \mathrm{s})=\quad \begin{array}{l}
V_{3}(z, s) \\
\operatorname{Ij}\{z, s)
\end{array}
\end{aligned}
$$

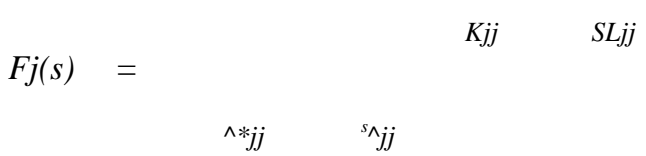

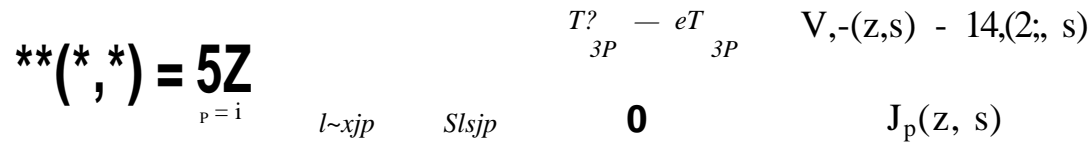

Here, $\operatorname{Vj}(z, s)=J^{r}\{v j(z, £)\}$ and $\operatorname{Ij}(z, s)=J^{7}\{i j(z, t)\}$ where $T\{$.$\} is the Fourier trans-$ form operator. Now, the equation (3.7) for $j^{t h}$ line can be decoupled and expressed as a recursive set of ODEs, by applying waveform relaxation [28]. The $(r+1)$ iteration of this relation can be written as

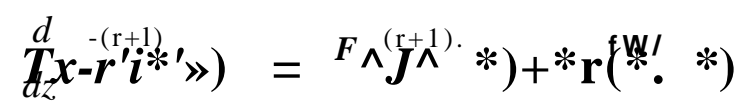

where $\wedge^{\prime}(z, s)^{r}$ is calculated from (3.5), using values of voltages and currents from the previous iteration (r). Solving the inhomogeneous ODE in (3.11), we get the following expression for the WR sources:

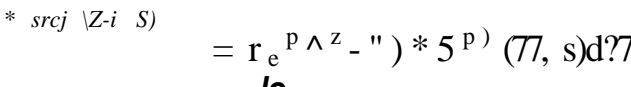

$$
\begin{aligned}
& *_{s r c j}[\% \bullet>S) \\
& \text { Jo }
\end{aligned}
$$


The relaxation sources (in (3.12)) are calculated by evaluating the convolution integral numerically, using a suitable integration formula. Integration formulas approximate the integral in (3.12) using a weighted sum of the form

$$
I^{l \cdot Z} \quad e^{F \wedge_{-} \wedge_{j}\left({ }_{v}, s\right) d r}=J 2^{w} k^{e F j / z} \sim^{Z k)} * 3(z k, s) A z_{k}
$$

where $W k$ is the weighting factor, $m$ is the number of discretization points, and $\mathrm{Az}_{\mathrm{fe}}=$ $Z k-Z(k-1)$. These frequency-domain sources(in (3.13)) are transformed into the time domain using IFFT so that the resulting time-domain relaxation sources can be connected to the end of each line, as shown in Figure 3.1. These sources are referred to as the single-ended sources. Note that in the circuit implementation of these relaxation sources, piecewise linear (PWL) source option available in SPICE-like simulators can be used.

The single-ended representation of the relaxation sources requires evaluation of

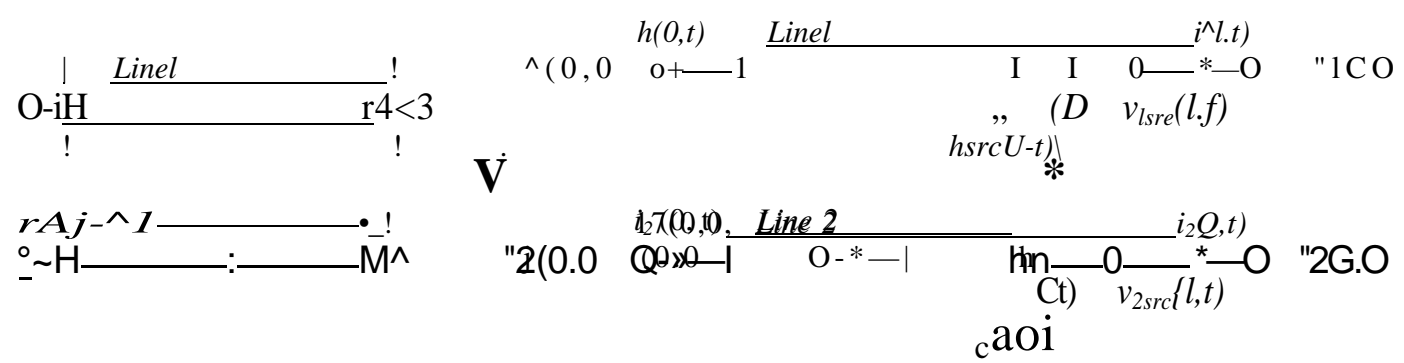

Figure 3.1: Transverse partitioning using single-ended sources. 
the convolution integral and time/frequency conversions at every iteration, making this method computationally expensive and less robust. A more efficient method that uses time-domain distributed sources and lumped segmentation was proposed in [6]. The key idea of using distributed sources is to represent the weighted sum using time-domain sources that are connected to each lumped segment of the line, instead of explicitly calculating the convolution integral in (3.13). The distributed sources for the $k^{\text {th }}$ section can be calculated as [6]

$$
\sim e f\left\{z_{k}, t\right)=-w_{k} J 2 \quad\left(\& Z k R_{3 p} i^{i} ;+A z_{k} L_{j p}-\int f \mathrm{~J}\right.
$$

$$
\left.\left.t \wedge\left(z_{k}, \quad t\right)=-w_{k} J 2 U z k G^{\wedge}-\langle>)+\mathrm{Az}_{\mathrm{fc}} \mathrm{C},{ }_{\mathrm{p}} \mid(\wedge-\text { if })\right)\right)
$$

Here $i j\left(z_{k}, t\right), q j\left(z_{k}, t\right)$ are the voltage and current sources, respectively. $V j, \quad v_{p}, i j$, $i_{p}$ are the voltages and currents of the $k^{\text {th }}$ section, from the previous iteration. The circuit realization of the distributed sources approach is shown in Figure 3.2. 


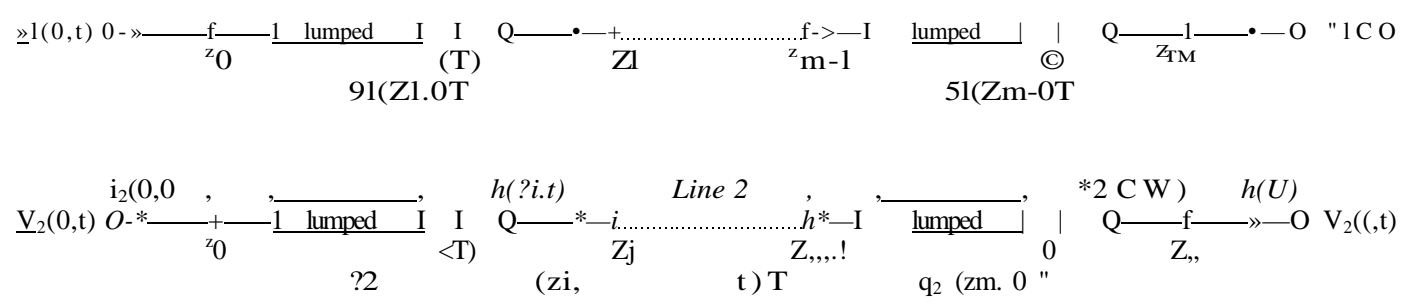

Figure 3.2: Transverse partitioning using distributed sources.

\subsection{Waveform Relaxation Method for DEPACT macromodel}

In the previous section, WR-TP was used to decouple the MTL into single lines. These single lines were modeled using the conventional lumped segmentation macromodel. However, the lumped segmentation model requires a large number of sections to accurately model electrically long lines, as indicated by the formula (2.33). These long lines can be represented more efficiently using the DEPACT macromodel, which was reviewed in Chapter 2. The DEPACT macromodel requires fewer sections and also guarantees the passivity of the resulting model. However, using the DEPACT algorithm directly on massively coupled interconnects result in large coupled circuits. As we saw earlier in Chapter 2, simulation of coupled circuits can be computationally expensive when the number of lines are large. To address this massive coupling, transverse partitioning of the DEPACT macromodel using WR was proposed in [29], 
and this method achieved a linear growth in CPU time with respect to the number of lines. Next, we review this WR based DEPACT macromodel. The transverse partitioning and calculation of relaxation sources for the DEPACT sections, namely, lossy section and lossless section of the DEPACT macromodel are considered next.

\subsubsection{Transverse Partitioning of DEPACT Lossy Sections}

For a DEPACT lossy section, the terminal voltages and currents can be written using the matrix-exponential stamp [30] as

$$
v\left(l_{m}, t\right) \quad=e^{A l,} \quad \begin{aligned}
& v\left(l_{0}, t\right) \\
& i\left(l_{0}, t\right)
\end{aligned}
$$

where $l_{m}$ is the length of one section; $v\left(l_{m}, t\right), i\left(l_{m}, t\right)$ and $v(l o, t), i(l o, t)$ are the voltages and currents at the near end and far end of the section, respectively. This relation is in the form of the T-parameters denned for a linear multi-port network.

$$
\begin{array}{llll}
v\left(l_{m}, t\right) & T n & T 12 & v\left(l_{0}, t\right) \\
& T 21 & T 22 & i\{l o, t)
\end{array}
$$


The $\mathrm{T}$-parameters can be converted to the $\mathrm{Y}$-parameters using the relation given below.

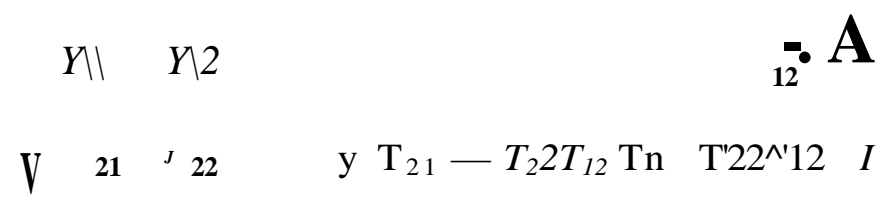

Therefore, the terminal voltages and currents of the section can be written as

$\begin{array}{llll}i(l o, t) & \wedge 11 & \wedge 12 & v\left(l_{0}, t\right) \\ & \wedge " 21 & \wedge 22 J & v\left(l_{m}, t\right.\end{array}$

Equation (3.19) can be re-arranged to group together the terminal voltages and currents of the same line

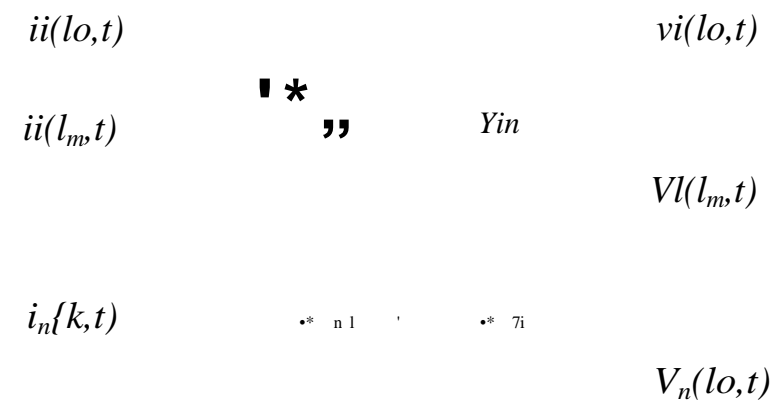

where $Y$ is the rearranged $\mathrm{Y}$-parameter matrix, and the off-diagonal terms $\mathrm{Y}_{\mathrm{JP}}$ represent the coupling between the lines. From (3.20), a closed-form formula for the WR 
sources can be derived as [29]

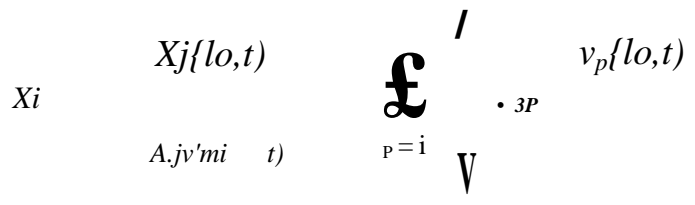

Here, the WR source $X j{ }^{\text {can }}$ be represented as current sources at the near and the far ends of a DEPACT lossy section. The resulting decoupled lossy sections are shown in Figure 3.3.

\subsubsection{Transverse Partitioning of DEPACT Lossless Sections}

The DEPACT lossless sections are decoupled using single-ended relaxation sources, discussed in Section 3.2. Note that the single-ended sources in the case of DEPACT lossless section result in closed-form time-domain sources (unlike the lumped segmentation model that required the numerical evaluation of a convolution integral). A closed-form formula for the time-domain WR source was derived in [29], for a coupled

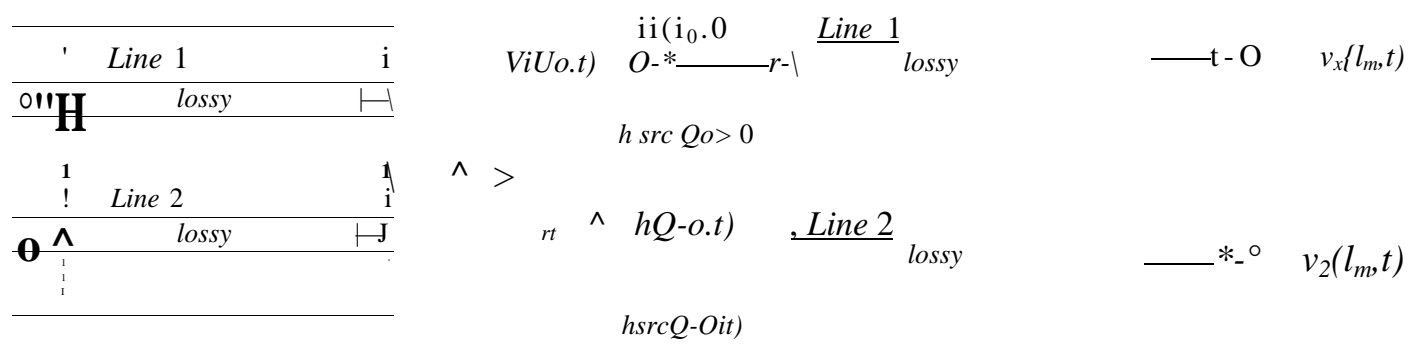

Figure 3.3: Decoupled DEPACT lossy section. 
n-line lossless section. The essential details of the derivation are reviewed here.

We recall from Section 3.2 that the single-ended relaxation sources can be defined in the frequency domain as a convolution integral

*j STCZ-I S)

$$
\text { Jo }
$$

In the case of lossless section, (3.22) can be simplified as

$$
\left.\left.\varlimsup_{J O} e^{F_{\wedge} l m, r}>\wedge_{j}(r], s\right) d r\right]
$$

where

$$
\begin{aligned}
& \mathrm{F} ;(\mathrm{s})=\begin{array}{cc}
0 & -s L_{33} \\
-s C_{J 3} & 0
\end{array}
\end{aligned}
$$

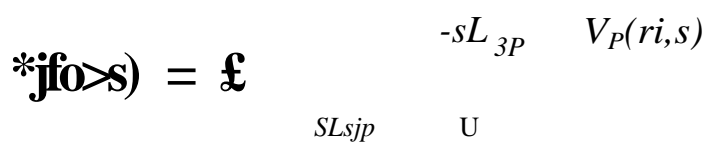


Next, define a vector $W_{n}(s)$ containing voltages and currents of the lines at position 77

$$
\left.W_{v}(s)=V_{n}(r), s\right)
$$

$$
I_{n}\left(v,{ }^{s}\right)
$$

and $W Q$ is the near-end voltages and currents at $77=\mathrm{Z}_{0}$. The voltage and current of $p^{\text {th }}$ line can be selected from $W_{v}(s)$ using a selector matrix $X J_{P}$ as

$$
=l J_{P} W_{v}(s)
$$

$$
I_{P}\left(V,{ }^{s}\right)
$$

where elements $U_{P i}$. $€\{0,1\}$. Then, $\wedge f j(s)$ can be written as

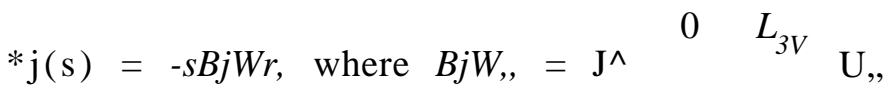

$$
\begin{aligned}
& \begin{array}{lll}
\stackrel{P}{=} \backslash \\
V+3
\end{array} \quad C j_{P} \quad 0
\end{aligned}
$$

Next, the voltages and currents of the lines are expressed in terms of near-end voltages and currents of the lossless section $W Q(S)$, using the matrix exponential 
stamp of the line.

$$
\begin{array}{cc}
\mathrm{O} & -L \\
-C & \mathrm{O}
\end{array}
$$

Using the equations (3.23)-(3.29) the WR sources can be written as

$$
\left.f_{\text {Jo }}^{m} \mathrm{e}^{\wedge \wedge}{ }^{\wedge} \mathrm{Bj}^{-} \mathrm{e}^{* 6 "} W_{0}(s) d r\right]
$$

The exponential matrices in (3.31) can be diagonalized as

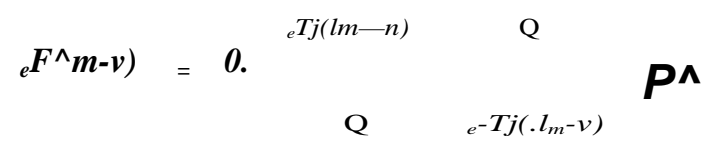


where $13 \cdot$ is the eigenvector of $F j / s$ and $T j=y / L j j C j j$, and

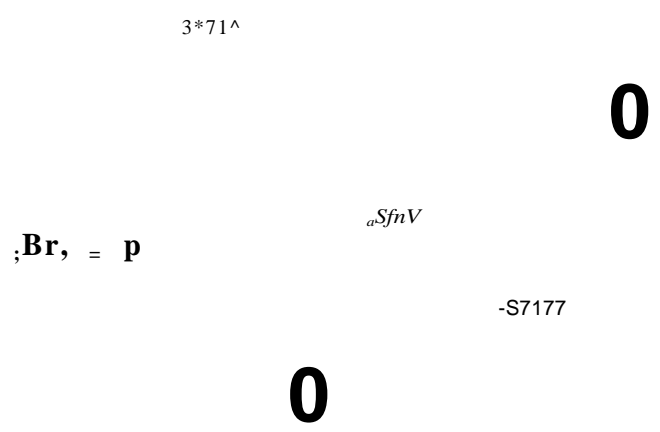

$7 \mathrm{i} . .-7 \mathrm{n}$ and $\mathrm{T}$ are the eigenvalues and eigenvectors of $\mathrm{B}$, respectively. Next, substituting (3.32) and (3.33) in (3.31) and evaluating the integral, we get 
where $H j$ is a $2 \times$ In matrix whose elements are defined as

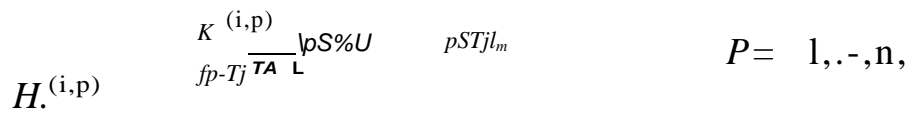

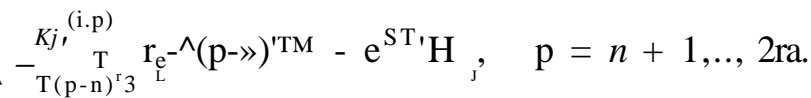

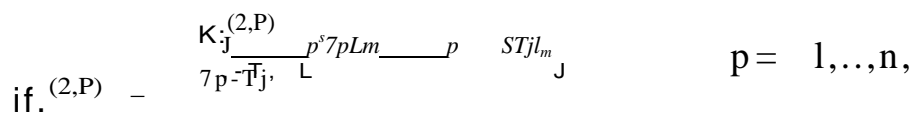

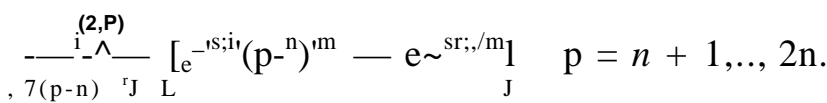

$$
\begin{aligned}
& \mathrm{K} \wedge \wedge \mathrm{B} \mathrm{f}
\end{aligned}
$$

Next, the time domain equivalent for these WR sources can be obtained by representing the exponential terms in (3.35) as time delays. That is, the WR sources can be obtained as a function of time-delayed near-end waveforms. We define a vector

$$
{ }^{*}\left({ }^{*}\right)=\underset{02 \mathrm{n}(*)}{\text { f } \boldsymbol{W}_{\boldsymbol{o}}(\boldsymbol{t})}
$$

where $\mathrm{W}_{0}(\mathrm{i})$ is the vector of near-end voltages and currents in time domain. Then, the closed-form formulas for time-domain WR sources can be written as

$$
\begin{array}{lllll}
* j & s r c y^{\wedge} m t & \mathrm{v} & -P i \\
*_{j} & s r c \backslash<m i & t)
\end{array}
$$


where

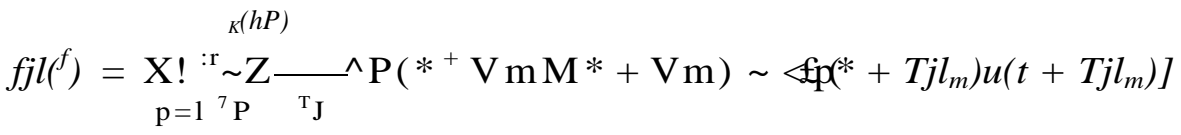

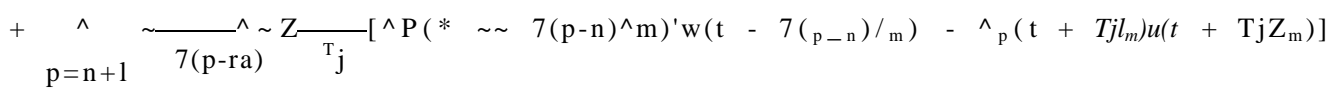

$$
\begin{aligned}
& / \mathrm{j} 2(*)=\underset{\mathrm{p}=1}{\|} \underset{{ }^{7} \mathrm{P}}{\sim} \mathrm{K}^{(2, \mathrm{p})}{ }_{{ }^{\mathrm{T}} \mathrm{J}}\left[\wedge \mathrm{P}\left(*+\%{ }^{l} \mathrm{~m}\right) u\left(t+7 \mathrm{p}^{\wedge} \mathrm{m}\right)-0 \mathrm{p}\left(*-T j l_{m}\right) u\left(t-\mathrm{TjZ}_{\mathrm{m}}\right)\right] \\
& \text { 2n } \quad-\quad J\left(\left(^{2} P\right)\right. \\
& \left.\left.+\underset{p=n+l}{\mathrm{X}]} \underset{\left.{ }^{7}<\mathrm{P}-"\right)}{\sim}{ }_{\wedge}[\wedge \mathrm{P}(* \quad \sim \quad \%-n) l m) u(t-\%-n) l m\right) \quad \sim \quad 4>p\left(t \quad \sim \quad T j l_{m}\right) u(t-T j l m)\right]
\end{aligned}
$$

where $u(t)$ is the unit step function. The resulting decoupled lossless sections are shown in Figure 3.4

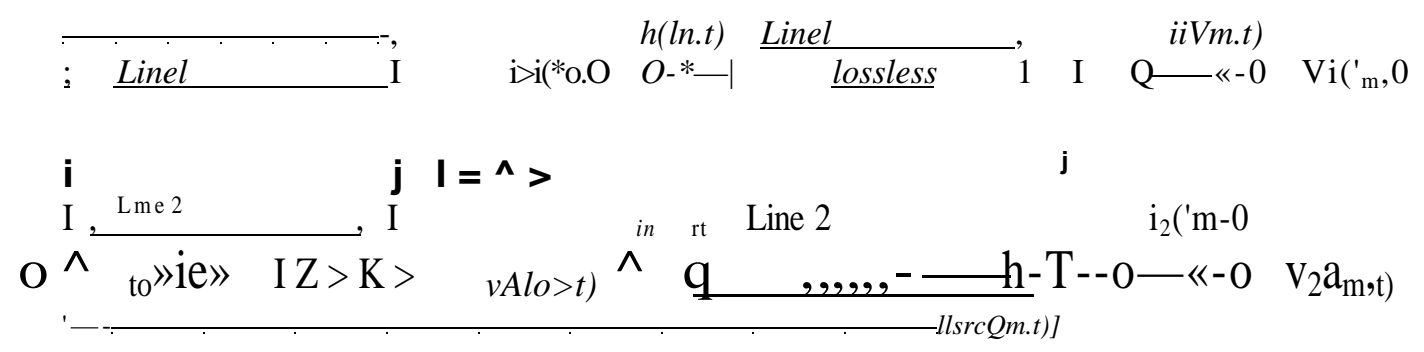

Figure 3.4: Decoupled DEPACT lossless section. 


\subsection{Summary}

In this chapter, the transient simulation of interconnect circuits using the waveform relaxation method was reviewed and its merits over the conventional methods were highlighted. Application of the waveform relaxation and transverse partitioning (WRTP) to coupled interconnects was reviewed. Also, it was shown that the distributed relaxation source approach lead to a time-domain representation of the WR sources, which is suitable for SPICE-like nonlinear simulators. Finally, a DEPACT-based macromodel using WR-TP that is suitable for long interconnects was reviewed. In the next chapter, we use these concepts presented to derive a new EMI macromodel that can efficiently handle large interconnect networks having long delays. 


\section{Chapter 4}

\section{EMI Analysis of Massively}

\section{Coupled Interconnects with Long}

\section{Delay}

As highlighted in Chapter 2, the conventional methods for EMI analysis of MTLs result in large coupled system of equations that cannot be efficiently solved using direct solvers. To solve such massively coupled interconnect circuits, the WR-TP method was proposed [6], which was reviewed in Chapter 3. Using WR-TP, a faster EMI analysis method for massively coupled interconnects was proposed in [8]. Although, this method addressed the problem of massive coupling, its application is limited to relatively short lines because it is based on the lumped segmentation macromodel. 
Long lines can be modeled efficiently using delay-extraction based MTL macromodels like the DEPACT. A DEPACT-based EMI macromodel was proposed in $[5,19]$, however, this macromodel did not use any partitioning strategy. Hence, the large size of the resulting coupled system limited this method's application to small number of lines.

A new macromodel for EMI analysis that combines the advantages of the DEPACT macromodel and the recent advancements in waveform relaxation [29] is proposed in this chapter. This macromodel can be used to efficiently simulate massively coupled interconnects having long delays, which are encountered in practical problems. The proposed macromodel can be formulated using either the MLF-I or MLF-II structure of the DEPACT macromodel. Both these formulations are detailed in this chapter, as they differ slightly in accuracy and simulation efficiency, and could provide the user an option to tradeoff between speed and accuracy.

This chapter is organized as follows. In Section 4.1, the proposed method based on MLF-I is derived from the field coupling models discussed earlier. In section 4.2, a MLF-II formulation for the proposed method is derived. In Section 4.3, numerical examples are presented to demonstrate the speed and accuracy of the proposed method. 


\subsection{EMI Macromodel Based on DEPACT (MLF-I) Macromodel}

EMI effects on interconnects can be analyzed by modeling these effects as field coupling to a MTL, represented using the inhomogeneous Telegraphers's equations [2], as discussed in Chapter 2. The solution of these equations can be written in the matrix exponential form given in (2.31). Next, to arrive at the proposed macromodel, we approximate the matrix exponential (2.31) in the time domain, using the MLF-I based DEPACT macromodel. Next, we find the equivalent distributed EMI sources to represent the convolution integral in (2.30). Finally, to obtain the proposed macromodel, we decouple the individual lossy and lossless sections of the DEPACT macromodel using WR-TP.

\subsubsection{Derivation of the Distributed Sources}

The matrix exponential in (2.31) can be approximated as cascade of $m$ MLF-I DEPACT sections. Each DEPACT section consists of one lossless delay element and two lossy lumped resistive sections. The $k^{\text {th }}$ DEPACT section for the case of a single line is shown in Figure 4.1.

Field coupling to each section is approximated as the integral (in (2.30)) evaluated for a lossless line of length - The voltages at the terminals of the lossless section, 
resulting from the scattered fields, are marked as $V f^{\ominus 1}, V^{\wedge 1}$, and the total currents at the terminals are marked as $\Lambda, 12$ - These voltages and currents at the terminals of the lossless section can be written using the equations derived for field coupling to MTLs (in 2.29) as

$$
\begin{gathered}
\frac{\mid T S C t}{\mathbf{v}_{2}} \\
\text { II }
\end{gathered}
$$

where $F(z, s)$ is the forcing function defined in (2.21) as

$$
F(z, s)=e^{s \wedge} T(s)
$$

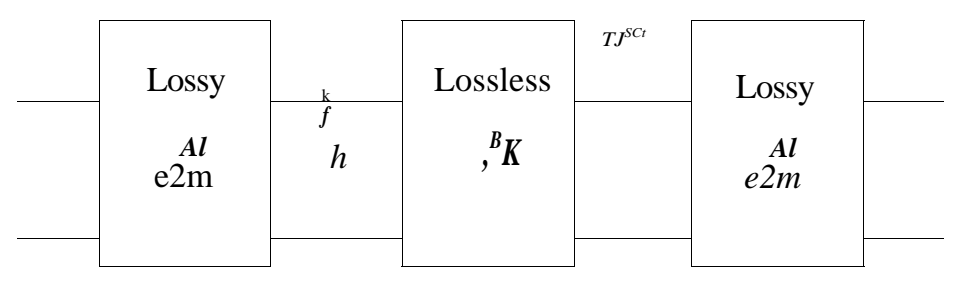

Figure 4.1: $k^{\text {th }}$ DEPACT section. 
and $z \backslash$ and $z_{2}$ are the near and far end positions of the $k^{\text {th }}$ section.

$$
\begin{aligned}
& z \backslash=k \bar{m} \\
& z_{2}=\{k+1) \bar{m}
\end{aligned}
$$

The integral in (4.1) can be evaluated as

$$
\begin{aligned}
& { }_{e} s B z_{2} \quad f^{2}{ }^{-\left(s B+V_{2 n X 2 n S K 2}\right) z} \quad \mathrm{r}(\mathrm{s}) \mathrm{d} z \\
& J z \mid
\end{aligned}
$$

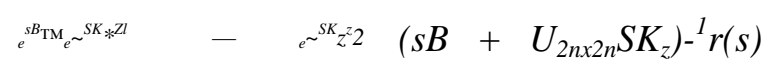

Substituting (4.5) in (4.1) and collecting the e $\mathbf{B}_{\Gamma} \dot{d}$ terms together, we get

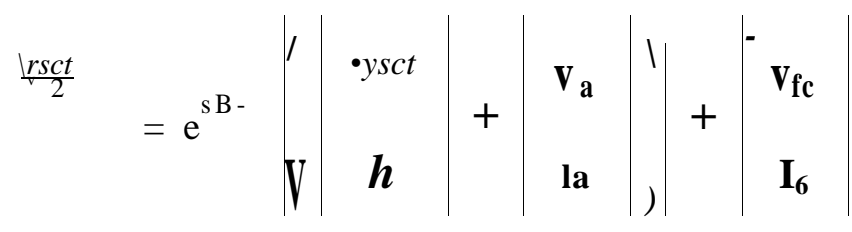

where $\mathrm{V}_{\mathrm{a}}, \mathrm{Vj}, \mathrm{I}_{\mathrm{a}}$, I\& are EMI sources connected to the terminals of each DEPACT section, which are defined as

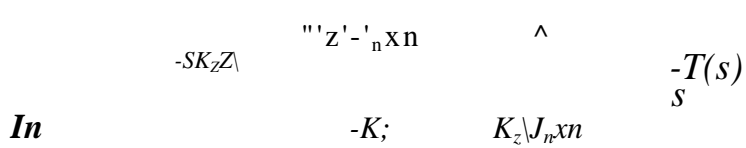

$$
\begin{aligned}
& -e^{-\underline{S} K_{2} z l} E_{0} c p
\end{aligned}
$$


Here, ( $p$ is independent of $s$, and is defined as

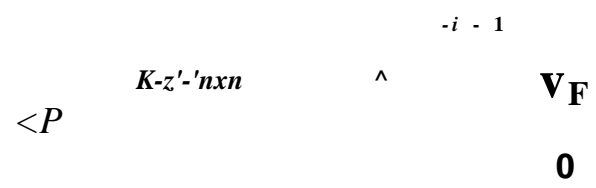

Similarly the far-end sources can be written as

$$
\mathrm{V}_{\mathrm{fc}} \quad \mathbf{e} \quad{ }^{\prime} E o<p
$$

It is to be noted that the complex exponential in (4.8) and (4.10) can be represented as delays in the time domain. Therefore, the closed-form formulas can be written for equivalent time-domain EMI sources, in terms of the time-delayed envelope of the electric field $E_{0}$. The sources for the $k^{\text {th }}$ section can be written as

$$
\begin{gathered}
E_{Q}\left(t-K_{z} \frac{k-}{m}\right) i p \\
-E_{0}\left(t-K_{z}(k+l) \frac{m}{m}\right)(p \\
\operatorname{vj}^{\prime \prime c}(0)=-2 E^{\operatorname{TM}^{c}}(t) V_{T} \\
v l^{n c}(l)=-2 E^{\operatorname{TM}^{c}}\left(t-n_{z} l\right) V_{T}
\end{gathered}
$$


Field coupling model based on the DEPACT (MLF-I) macromodel can be obtained by cascading $m$ DEPACT sections, along with the embedded distributed EMI sources

defined in (4.11) and (4.12), and the terminal sources defined in (4.13) and (4.14). The resulting coupled macromodel is shown in Figure 4.2.

The coupled DEPACT sections can be computationally expensive when the number of lines are large as in the case of high-speed buses. To address this issue, a decoupled macromodel based on waveform relaxation is proposed for EMI analysis involving massively coupled interconnects.

\subsubsection{Proposed Decoupled EMI Macromodel (WR-TP + MLF- I)}

The DEPACT sections can be decoupled into individual lines using WR-TP [29], by adding the appropriate WR sources to each section. The closed-form formulas of these WR sources defined for the lossy and lossless DEPACT sections can be obtained as detailed in Sections 3.3.1 and 3.3.2. The WR sources for the DEPACT sections are combined with the EMI sources while developing the proposed decoupled macromodel.

The steps required for EMI analysis using the proposed macromodel can be described as follows. First, using the geometry of the MTL and the incident wave parameters, the equivalent EMI sources defined in (4.11)-(4.14) are calculated. It is 

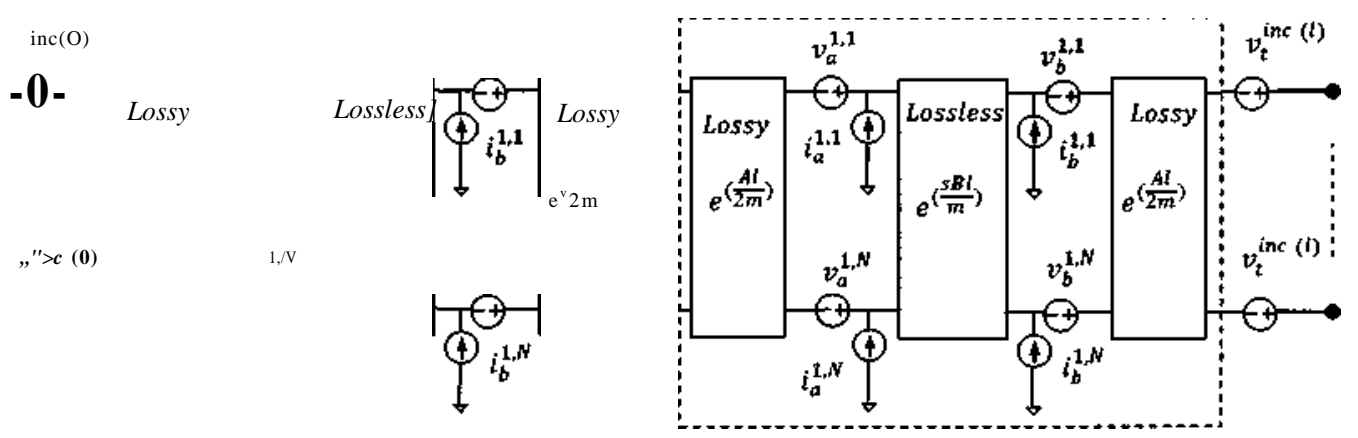

Figure 4.2: Coupled macromodel based on MLF-I

to be noted that if the EMI sources for the first section are calculated, sources for the other sections are just time delayed versions of the these. Next, the MTLs are represented using the decoupled DEPACT sections (along with the corresponding WR sources) as shown in Figure 4.3. Although, the EMI sources and WR sources connected to individual sections are shown separately for clarity, these can be combined into one voltage and current source in the circuit realization. Once the equivalent circuit for the proposed macromodel is built, transient simulation can be performed.

Transient simulation is started by assuming an initial guess for all the WR sources (usually 0). The individual lines are simulated to obtain updated values of the terminal voltages at each DEPACT section. These updated values of voltages and currents are used to calculate the WR sources for the next iteration. The individual lines are again simulated with the updated values of the WR sources. This iterative process 
is repeated until the line voltages and currents converge within an acceptable error.

The above steps are summarized in Pseudocode 4.1.2. The final macromodel which has both the EMI sources and WR sources is shown in Figure 4.3.

Pseudocode 4.1 Proposed EMI analysis algorithm (MLF-I)

Subdivide the coupled interconnect into $n$ lines using WR-TP.

Represent each line using DEPACT sections

Calculate the EMI sources for the first section using (4.11)-(4.12) and then using appropriate time delays obtain sources for the rest of the sections

$i<-1$ iteration $\}$

5 while e $>$ TOL or $i<$ iter MAX do

$6 \quad$ if $i=1$ then

$7 \quad$ Assume $V s_{r c}=0, i_{s r c}=0$ \{initial guess for WR sources $\}$

8 else

9 Update WR sources using previous iteration solution $\mathrm{v}^{\wedge-}{ }^{1} \backslash \mathrm{i}\left(*^{*}{ }^{1}\right)$

10: $\quad$ end if

11: $\quad$ for $I-1$ to $n$ do \{loop of lines

12: Simulate the line / with EMI sources and WR sources, to obtain $\triangleright \mathrm{W}$,

13: $\quad$ end for

14: $\left.\quad \mathrm{e}=\operatorname{norm}\left(\mathrm{vW}-\mathbf{v}^{\wedge \prime \prime 1}, \mathbf{i} \ll-\mathbf{i}^{\wedge \prime \prime 1}\right)\right)$

15: $\quad i<-i+1$

16: end while 

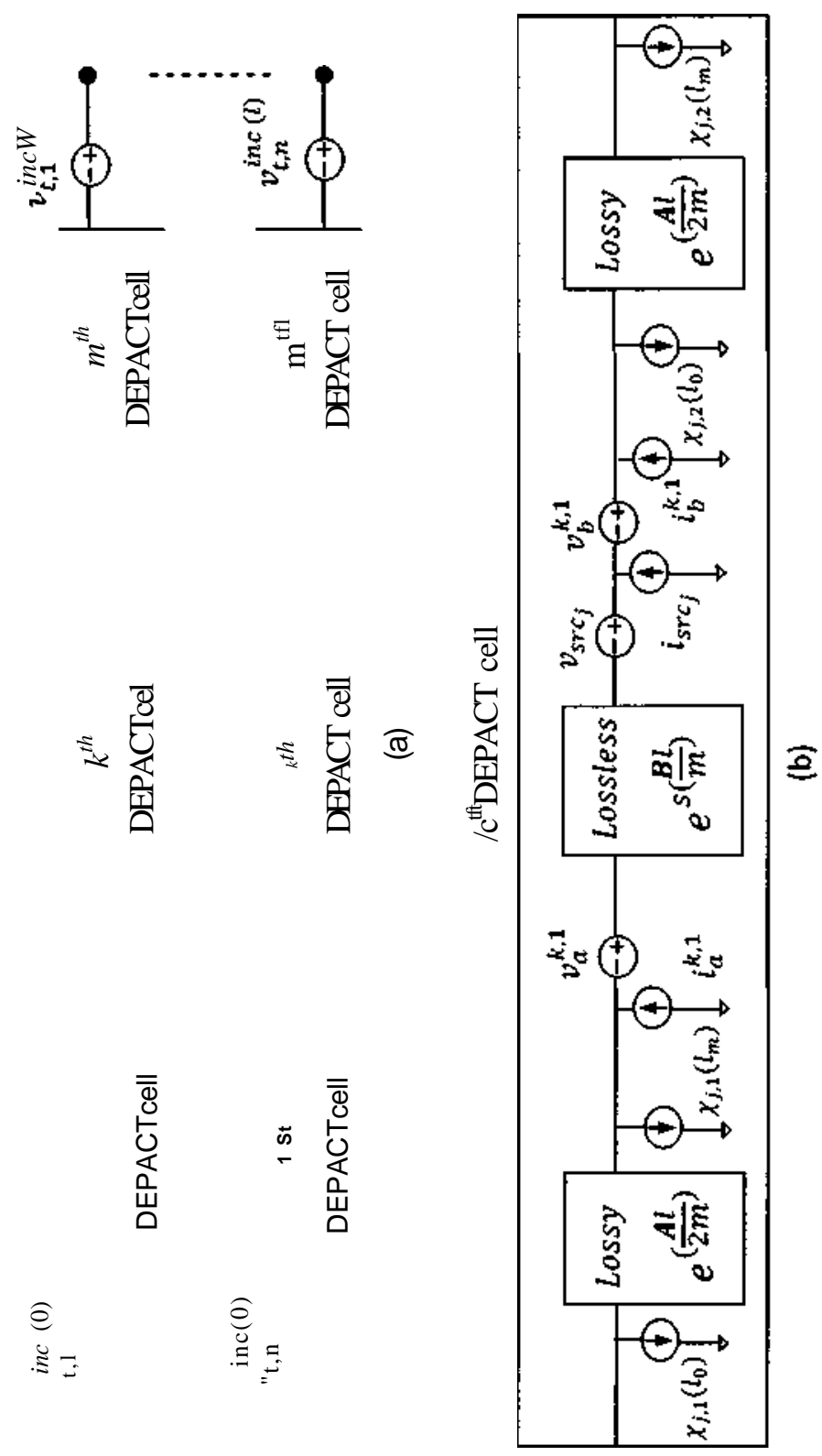

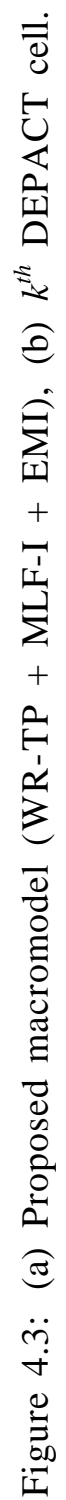




\subsection{EMI Macromodel Based on DEPACT (MLF-II) Macromodel}

An interconnect macromodel with better accuracy can be realized using MLF-II DEPACT sections [7]. A coupled macromodel based on MLF-II was derived in [5], which was reviewed in Chapter 2 (see Figure 2.9). However, for massively coupled lines this model can be inefficient because of the resulting large coupled system. Hence, a decoupled macromodel based on WR-TP is proposed [31].

\subsubsection{Proposed Decoupled EMI Macromodel (WR-TP + MLF-} II)

The decoupled MLF-II EMI macromodel is obtained by using similar steps as in the case of decoupled MLF-I model. That is, each MLF-II DEPACT section in the coupled model is decoupled using waveform relaxation to obtain individual lines with corresponding WR and EMI sources. Excluding the differences in the structure and the EMI sources, the steps required for transient simulation using MLF-II based macromodel are similar to the steps in MLF-I based model listed in Pseudocode 4.1.2. The final MLF-II based macromodel having the appropriate WR and EMI sources is shown in Figure 2.9. The corresponding EMI sources are defined in (2.48)-(2.50). 


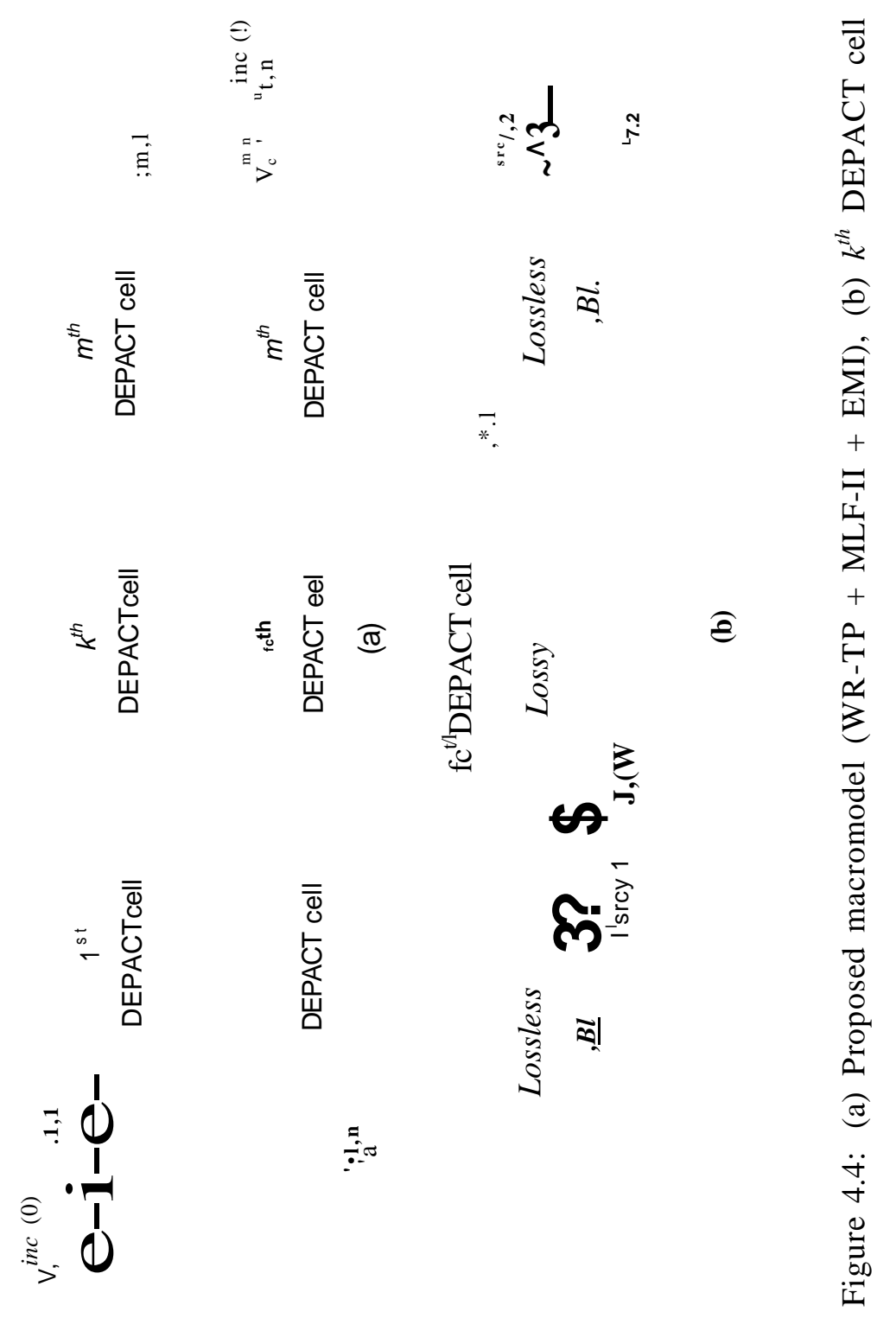




\subsection{Numerical Examples}

In this section, the proposed algorithms (both MLF-I and MLF-II based macromodels) are employed to simulate several interconnect structures that are excited by a plane wave as well as terminal sources. For the purpose of accuracy comparison, the simulation results are compared with the conventional EMI analysis that uses coupled lumped segment transmission line macromodel and time-domain EMI sources calculated using IFFT. In these experiments, the EMI sources are calculated with the assumption that a homogeneous dielectric medium is present all around the conductors.

Examples 1 and 2 demonstrate the validity and accuracy of the method while using linear and nonlinear terminations, respectively. Example 3 uses a large coupled interconnect structure having long delay, which demonstrates the CPU benefits of the proposed method. The proposed algorithm was implemented partly in Matlab, which was used to calculate the WR and EMI sources in each iteration. These sources (as PWL data) were fed to HSPICE, which was used to simulate the resulting interconnect subcircuits.

\section{Example 1: Two Lines Illuminated by a Plane Wave}

A two conductor transmission line with microstrip structure of length $2.5 \mathrm{~cm}$ (Figure 4.5) is considered in this example [32]. This structure is excited by a plane wave 
incident at the origin as shown in Figure 2.3, where $9=45^{\circ},<p=0^{\circ}$ and polarized at an angle $O E=-90^{\circ}$. The time envelope of the electric field was defined as a Gaussian pulse [8] of amplitude $l \mathrm{kV} / \mathrm{m}$ and pulse width of $0.1 \mathrm{~ns}$.

In the proposed method, the MTL structure was divided into single line subcircuits having appropriate WR and EMI sources. Each line was represented using the DEPACT macromodel with an approximation order of $m=4$. The simulation results of the proposed (WR-TP + MLF-I/II +EMI) models were compared with the results of conventional coupled lumped segmentation model [2] (shown in Figures 4.6 and 4.7). As seen from the graphs, the results obtained using the proposed MLF-I and MLF-II based EMI analysis models and conventional coupled model (using lumped segmentation) are in good agreement. It is to be noted that the proposed EMI analysis algorithm converged within $3 \mathrm{WR}$ iterations to obtain results comparable to the conventional analysis method. The difference between the conventional and proposed method results were measured at each iteration, and the corresponding 2-norm of the errors are presented in Table 4.1. 


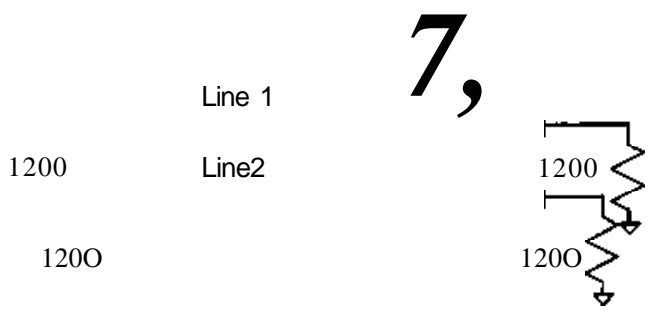

$2 \mathrm{~J}$,

$$
\begin{aligned}
& t \quad £ r=4.5 \\
& +\quad \tan S=0.001 \\
& \text { ¿ } \quad / \mathrm{i}=1194 \mathrm{fim} \\
& w=381 \mathrm{fim} \\
& S_{r} \quad d=1143 \mathrm{~nm} \\
& t-36 \text { fim } \\
& \text { Ground plane }
\end{aligned}
$$

w

w

Figure 4.5: Example 1: Structure of 2-line MTL.

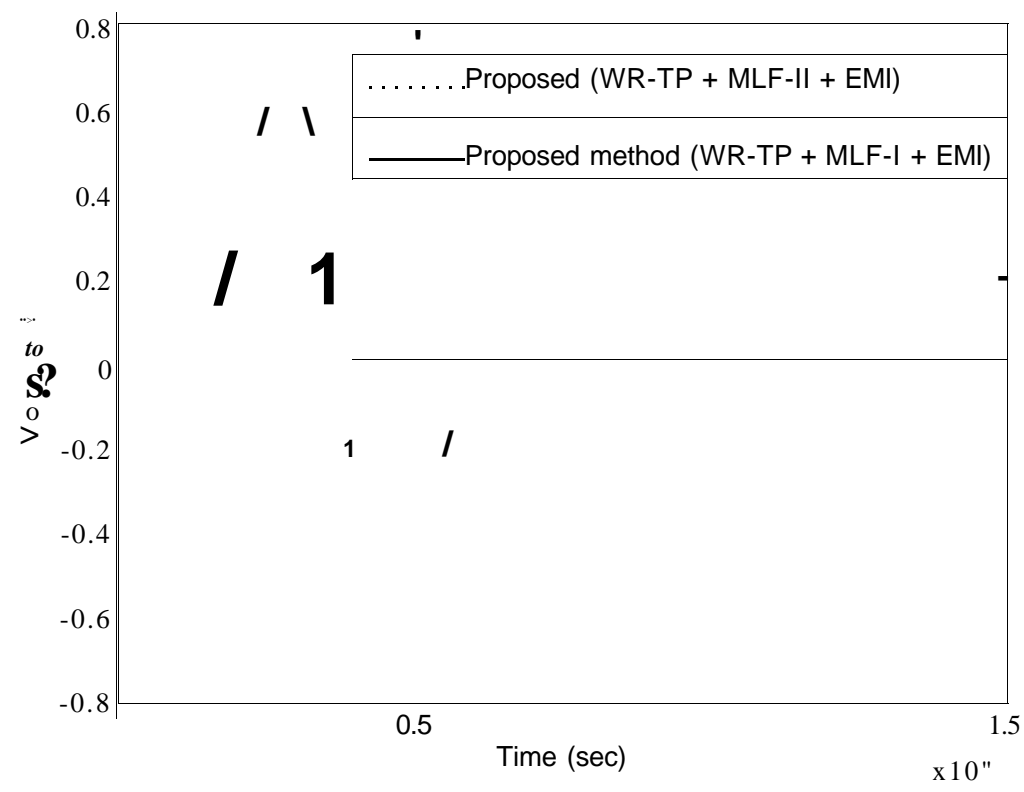

ure 4.6: Example 1: Comparison of transient responses at the far end of line 1. 


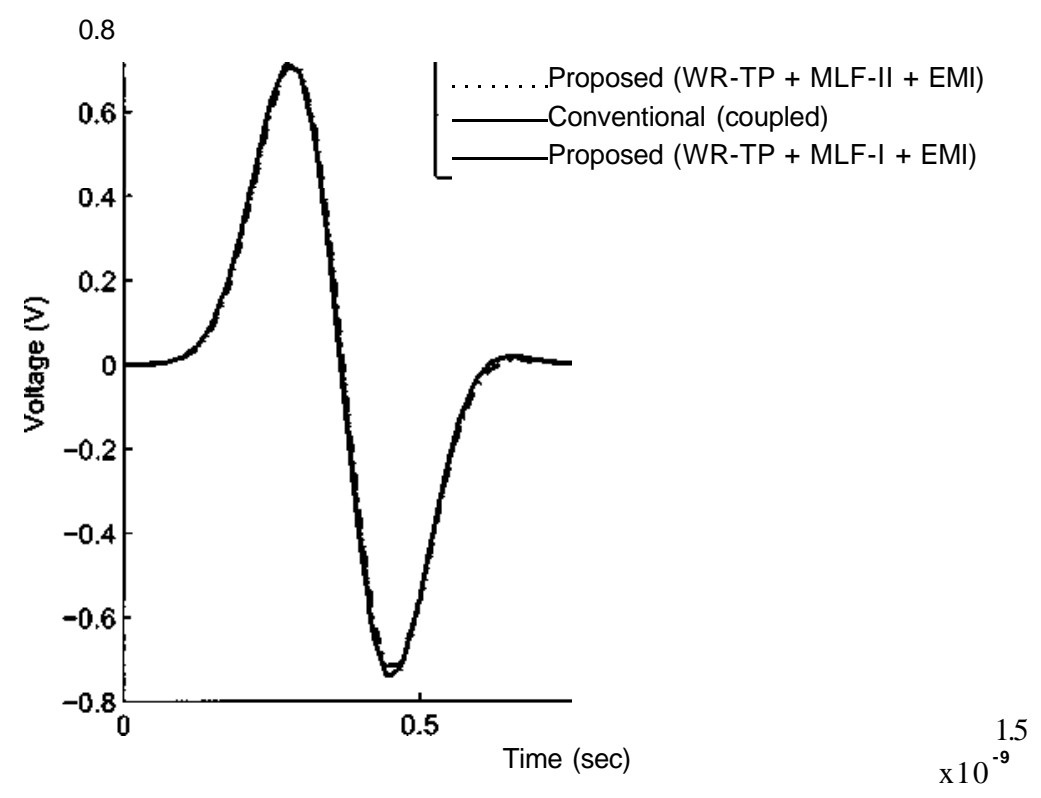

Figure 4.7: Example 1: Comparison of transient responses at the near end of line 2.

\begin{tabular}{|c|c|c|}
\hline Iteration & $\begin{array}{c}\text { Proposed method } \\
\text { (MLF-I based) } \\
\| \text { error } \\
end{array}$ & $\begin{array}{c}\text { Proposed method } \\
\text { (MLF-II based) } \\
\text { \|error } \\
end{array}$ \\
\hline 1 & 11.5 & 11.5 \\
\hline 2 & 0.187 & 0.146 \\
\hline 3 & 0.135 & 0.053 \\
\hline
\end{tabular}

Table 4.1: Example 1: Comparison of error norms for MLF-I and MLF-II based models. 


\section{Example 2: Lines Terminated by Nonlinear Devices}

In this example, we consider a nonlinear circuit containing a 3-line microstrip of length $30 \mathrm{~cm}$ (Figure 4.8). The Line 1 is connected to a CMOS inverter. These lines are excited by a plane wave incident at the origin as shown in Figure 2.3, where $9=45^{\circ}, 0=0^{\circ}$ and polarized at an angle $B E=-90^{\circ}$. The time envelope of the electric field was denned as a Gaussian pulse of amplitude $-500 \mathrm{~V} / \mathrm{m}$ and pulse width of $2 \mathrm{~ns}$. In addition, a trapezoidal pulse voltage source (rise/fall time Ins, amplitude $1.8 \mathrm{~V}$, pulse width $5 \mathrm{~ns}$ ) is connected to the near-end of the line through a series termination of $60 f 2$ as shown in Figure 4.8.

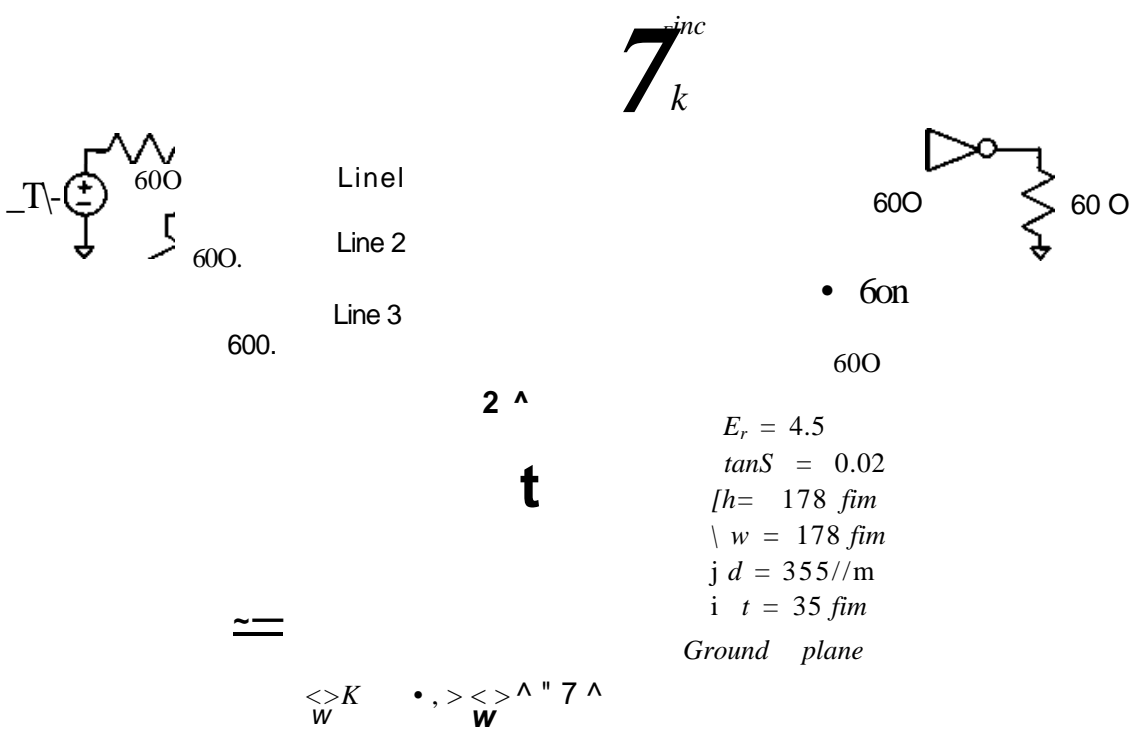

Figure 4.8: Example 2: Structure of 3-line MTL. 
The proposed method is used to simulate this interconnect. The results obtained after 3 iterations of WR are shown along with the conventional method results in Figures 4.9 and 4.10. The results from the proposed EMI analysis method (WR-TP + MLF-II) matches well with the conventional method (coupled lumped segmentation) [2] results, after 3 iterations. The difference between the conventional and proposed method results were measured at each iteration, and the corresponding 2-norm of the errors are presented in Table 4.2

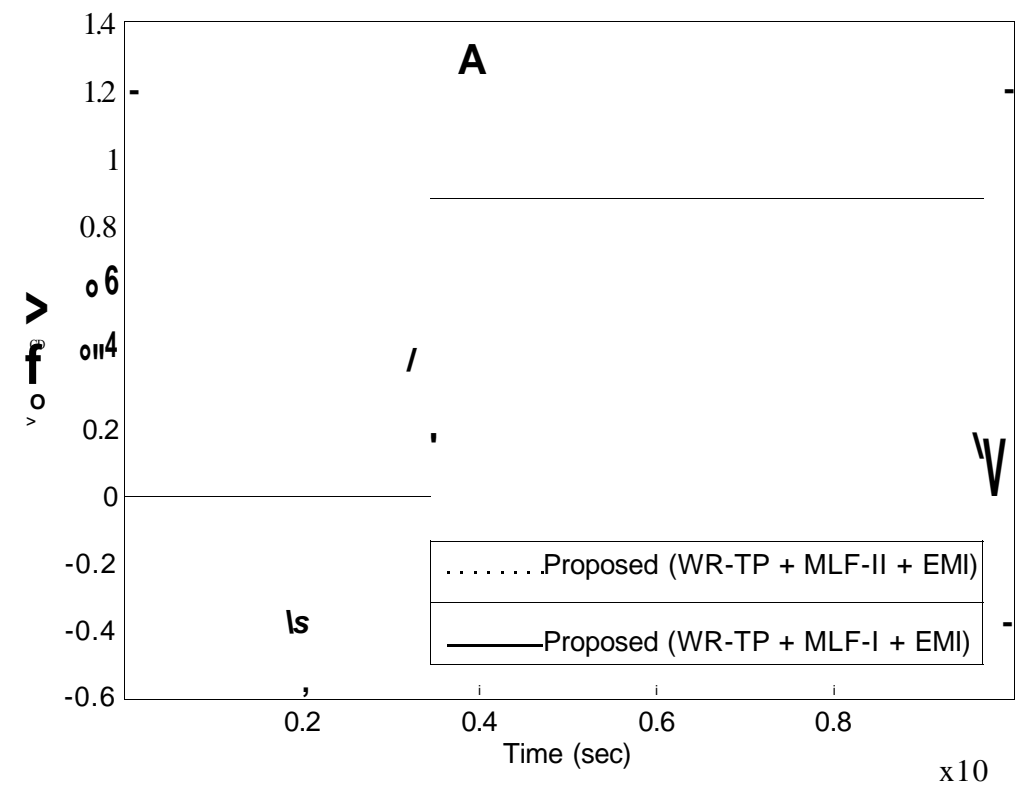

Figure 4.9: Example 2: Comparison of transient responses at the far end of line 1. 


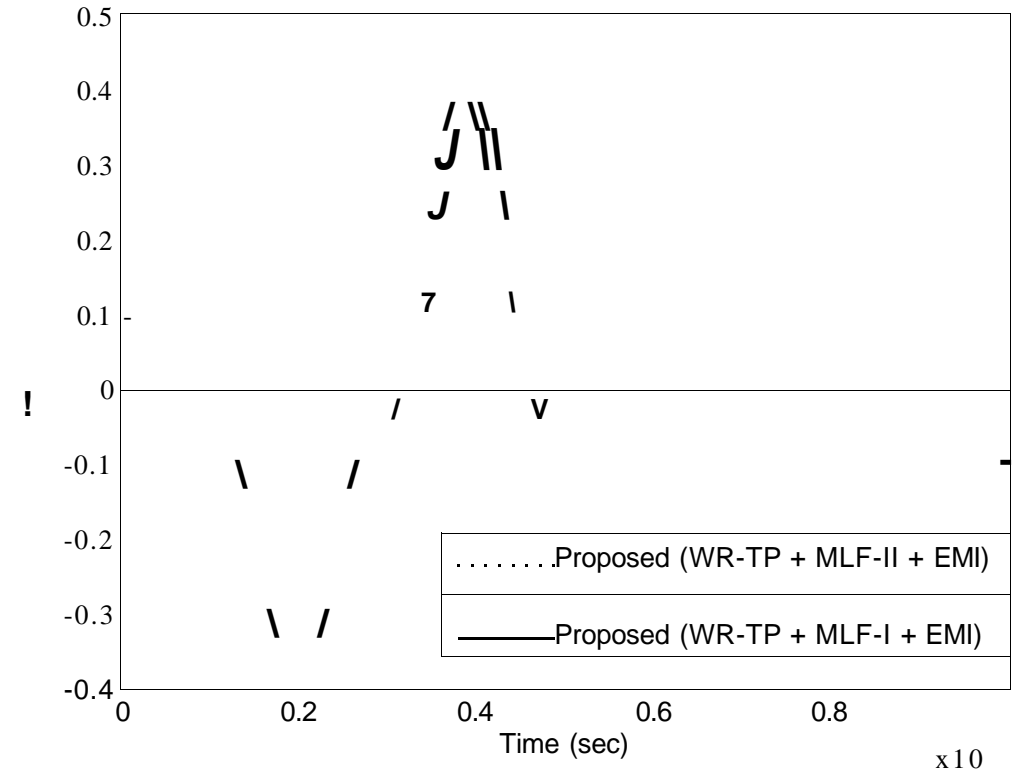

Figure 4.10: Example 2: Comparison of transient responses at the near end of line 3.

\begin{tabular}{|c|c|c|}
\hline Iteration & $\begin{array}{c}\text { Proposed method } \\
\text { (MLF-I based) } \\
\text { \|error } \\
end{array}$ & $\begin{array}{c}\text { Proposed method } \\
\text { (MLF-II based) } \\
\text { \|error } \\
end{array}$ \\
\hline 1 & 4.14 & 4.14 \\
\hline 2 & 0.331 & 0.064 \\
\hline 3 & 0.311 & 0.022 \\
\hline
\end{tabular}

Table 4.2: Example 2: Comparison of error norms for MLF-I and MLF-II based models. 


\section{Example 3: Large coupled interconnect}

A microstrip with 10 conductors, each of length $60 \mathrm{~cm}$ is considered in this example.

The cross-sectional geometry of the MTL is shown in Figure 4.11. The near and far ends of the MTL are terminated to ground using $50 f 2$ resistors and IpF capacitors, respectively. The line 1 is connected to a trapezoidal pulse current source. Also, this structure is excited using a plane wave, with electric field having a Gaussian temporal envelope with peak of $6000 \mathrm{~V} / \mathrm{m}$ and pulse width of $2 n s$.

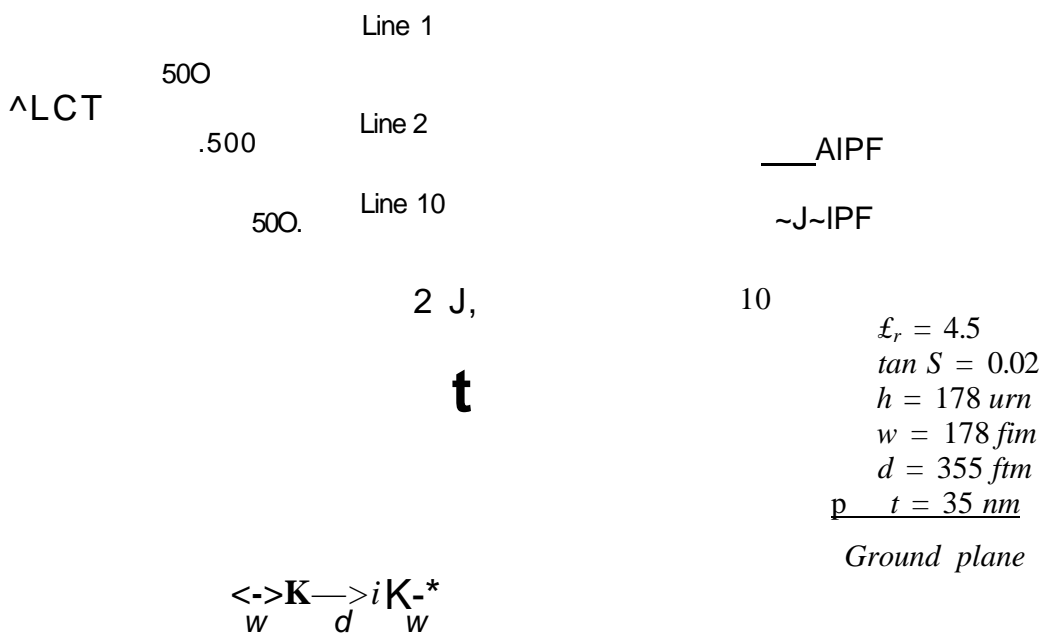

Figure 4.11: Example 3: Structure of 10-line MTL. 
Using the proposed method, this MTL was divided into 10 single line subcircuits having appropriate distributed WR and EMI sources. Each line was represented using the DEPACT macromodel (MLF-II) with an approximation order of $m=6$. Accuracy comparison of the simulation results from the proposed macromodel (WR$\mathrm{TP}+\mathrm{MLF}-\mathrm{II}+\mathrm{EMI}$ ) and the conventional method (coupled lumped segmentation) is given in Figure 4.12 and 4.13 (after 4 WR iterations).

In the next part of the experiment the scalability of the algorithms is investigated by measuring CPU times for varying number of lines. Table 4.3 provide the corresponding CPU times, and Figure 4.14 gives a graphical comparison of the CPU times of the proposed (WR-TP + MLF-II + EMI) and conventional method (coupled lumped segmentation [2]). Also, the CPU times of proposed method is compared with a recently published EMI analysis method (WR-TP + lumped segmentation + EMI [8]) in Table 4.4, and the corresponding graph is presented in Figure 4.15. As seen from the results, the proposed method scales well (almost linearly) with number of lines while yielding superior performance to both the existing methods. 


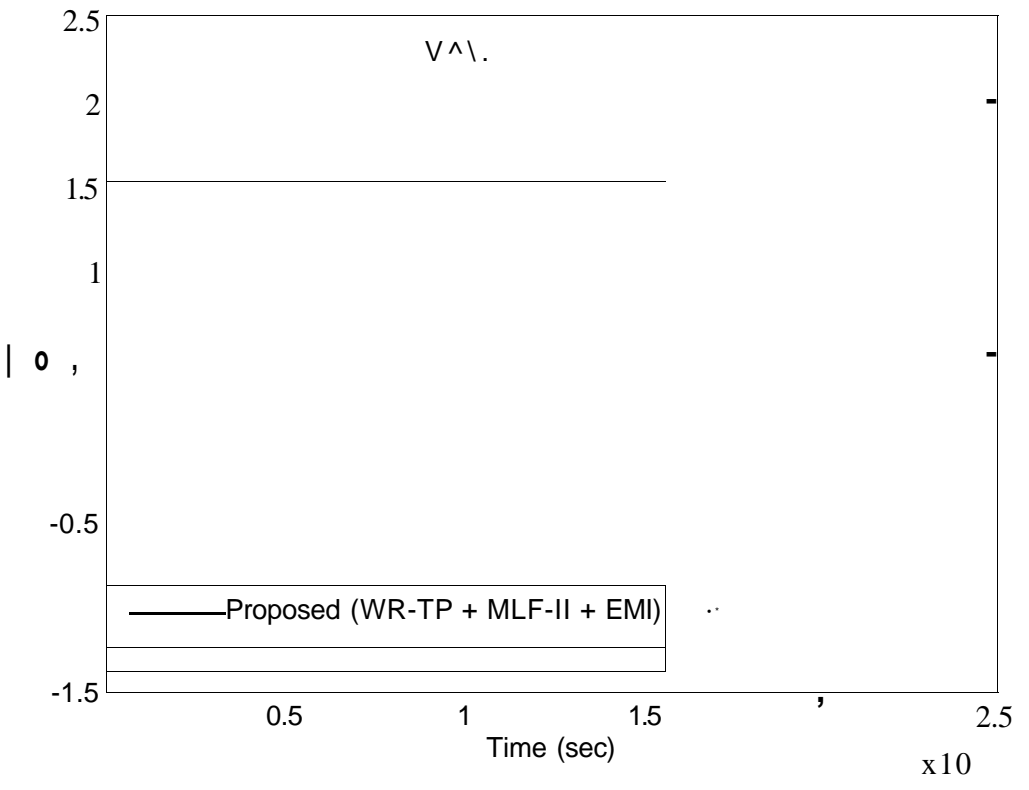

Figure 4.12: Example 3: Comparison of transient responses at the near end of line 1. 


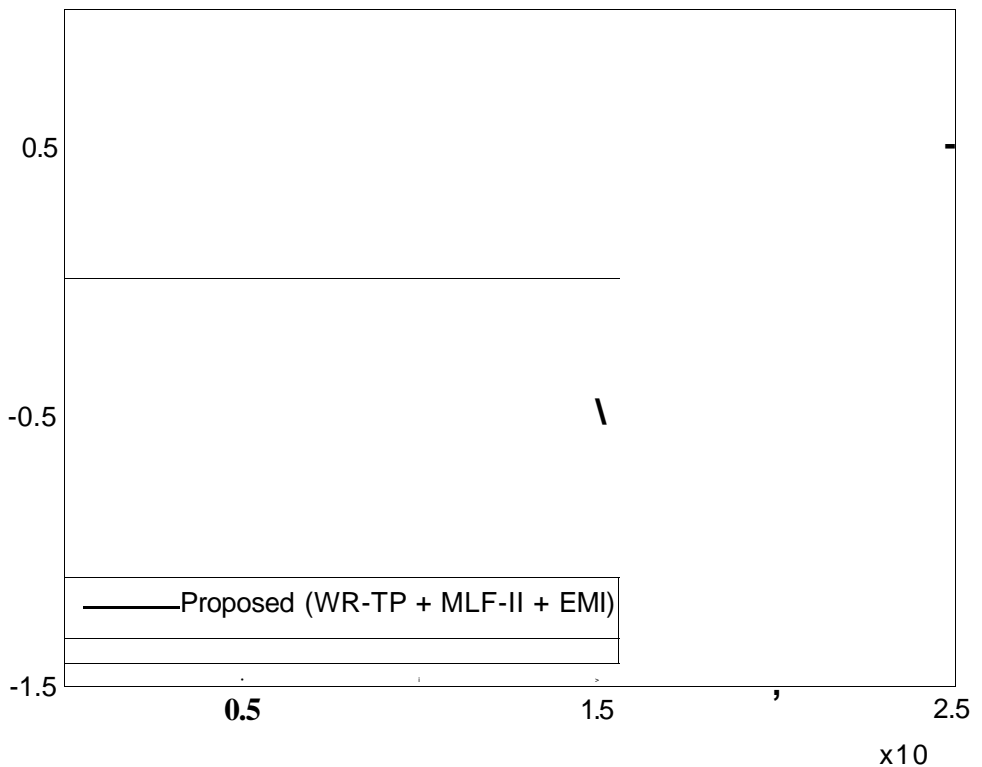

Figure 4.13: Example 3: Comparison of transient responses at the near end of line 10. 


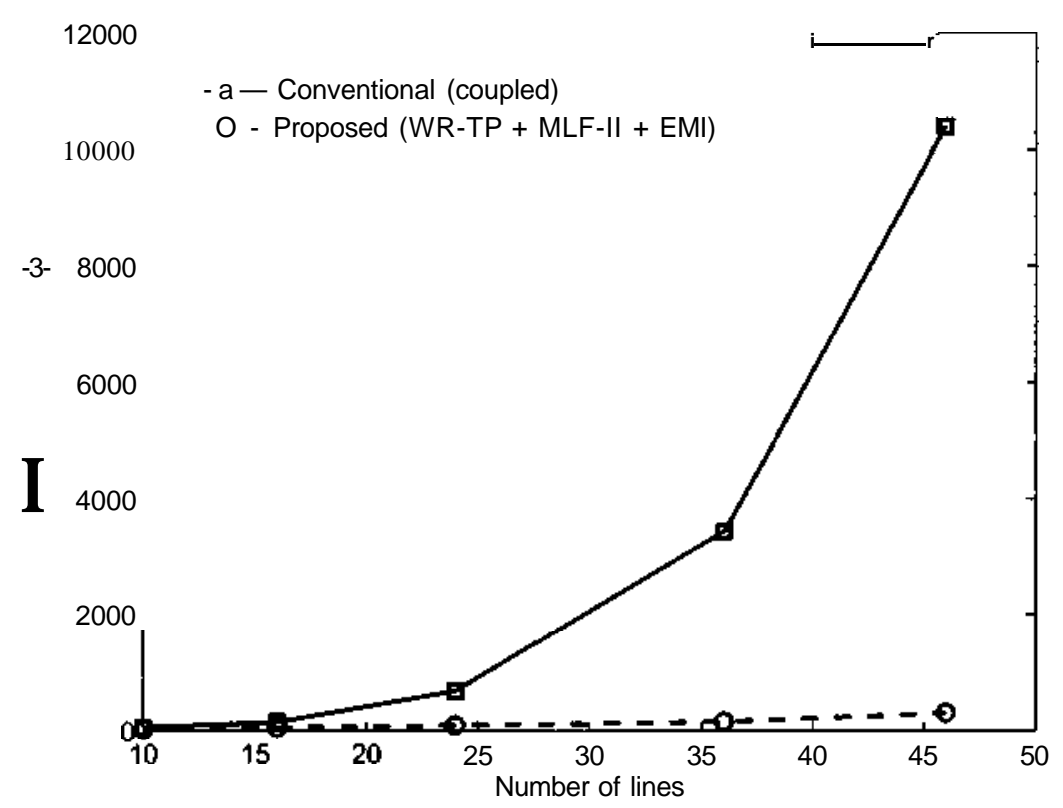

Figure 4.14: Example 3: Simulation time comparison of proposed EMI analysis method (WR-TP + MLF-II + EMI) vs conventional (coupled lumped segmentation) method.

\begin{tabular}{|c|c|c|c|}
\hline Number of lines & $\begin{array}{c}\text { Conventional (coupled) } \\
\text { simulation time (s) }\end{array}$ & $\begin{array}{c}\text { Proposed method } \\
\text { simulation time (s) }\end{array}$ & Speed-up \\
\hline 10 & 52.2 & 23.1 & 2.3 \\
\hline 16 & 139.6 & 47.5 & 2.9 \\
\hline 24 & 665.4 & 85.6 & 7.8 \\
\hline 36 & 3430.3 & 156.6 & 21.9 \\
\hline 46 & 10400.8 & 306.9 & 33.9 \\
\hline
\end{tabular}

Table 4.3: Example 3: Simulation time comparison of proposed EMI analysis method (WR-TP + MLF-II + EMI) vs conventional (coupled lumped segmentation) method. 
800
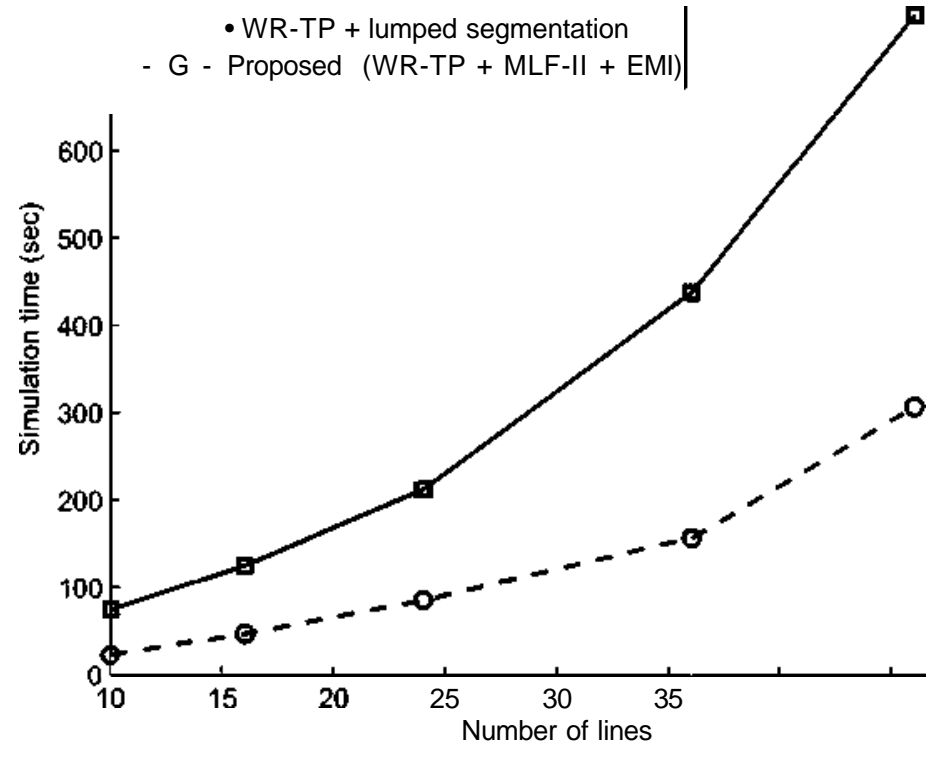

50

Figure 4.15: Example 3: Simulation time comparison of proposed EMI analysis method (WR-TP + MLF-II + EMI) vs existing (WR-TP + lumped segmentation + EMI) method.

\begin{tabular}{|c|c|c|c|}
\hline Number of lines & $\begin{array}{c}\text { WR-TP + lumped segmentation } \\
\text { simulation time (s) }\end{array}$ & $\begin{array}{c}\text { Proposed method } \\
\text { simulation time (s) }\end{array}$ & Speed-up \\
\hline 10 & 74.4 & 23.1 & 3.2 \\
\hline 16 & 124.9 & 47.5 & 2.6 \\
\hline 24 & 211.9 & 85.6 & 2.5 \\
\hline 36 & 437.9 & 155 & 2.8 \\
\hline 46 & 755.8 & 306.9 & 2.5 \\
\hline
\end{tabular}

Table 4.4: Example 3: Simulation time comparison of proposed EMI analysis method $(\mathrm{WR}-\mathrm{TP}+\mathrm{MLF}-\mathrm{II}+\mathrm{EMI}) v s$ existing (WR-TP + lumped segmentation + EMI) method. 


\subsection{Summary}

In this chapter a new macromodel for fast EMI analysis of large interconnects with long delay was proposed. This macromodel exploits the recently proposed WR-TP algorithm that can handle large coupled interconnects, and additionally uses the DEPACT macromodel to model long lines efficiently. The numerical examples showed that the proposed method achieves a linear growth in computational complexity $O(n)$, compared to $O\left(n^{4}\right)$ required by conventional method. In addition, the proposed method achieves a $2 \mathrm{X}-3 \mathrm{X}$ speed-up compared to the existing WR-TP based EMI analysis method. Next, a parallel algorithm for EMI analysis of interconnects will be presented. 


\section{Chapter 5}

\section{Parallel EMI Analysis of Massively}

\section{Coupled Interconnects}

Recently, computing platforms are experiencing a paradigm shift from uniprocessor based platforms to multiprocessor based platforms. The popularity of multicore architecture is evident from the recent surge in availability of multicore processors having dual, quad and even more cores. In addition to the speed advantage provided by the parallel hardware, parallel application development for multicore processors is greatly supported by the recent progress made in standardizing the programming environment, like OpenMP [33] that ensures quicker development and longer life cycle for the applications. Also, considering the large memory and processor requirements of interconnect simulation problem, it is a suitable application that can greatly benefit 
from parallel processing.

The parallel circuit simulation features offered by many vendors are limited to multi-threaded model evaluation [34], but the parallel simulation of the circuit (solving the MNA equations) has not been fully exploited. Moreover, interconnect simulation does not gain a noticeable advantage by speeding up just the model evaluation because of the large size of the corresponding circuit (mainly due to large number of nodes required to represent the distributed interconnect). Hence, parallelizing the simulation is important to gain any significant speed-up in the case of interconnect simulations.

Recently an EMI analysis algorithm suitable for parallel processing was proposed in [35], which uses physical partitioning based on WR-TP. However, this algorithm does not achieve an optimum processor utilization when the number of processors is not an integer multiple of number of lines. This can be deduced from the results in [9], where it was showed that just using the physical partitioning based on WRTP may not be sufficient to achieve 100 percent CPU utilization. To achieve better CPU utilization, time partitioning was suggested in the same paper and the resulting parallel simulation using physical and time partitioning (PS-PTP) algorithm showed a superior CPU utilization and speed-up characteristics with different numbers of processors. In the time partitioning scheme, the total time interval is subdivided into time blocks that can be simulated independently. 
In this thesis, the scope of PS-PTP [9] is extended for handling parallel EMI analysis of interconnects. The details of the proposed parallel algorithm and a brief review of background material is presented in this chapter. In Section 5.1, the EMI analysis method based on physical partitioning is reviewed. In Section 5.2, the details of the proposed algorithm are presented. In Section 5.3, numerical examples and the simulation speed measurements are presented.

\subsection{Review of EMI Analysis of Interconnects Us- ing Physical Partitioning}

Partitioning of the computation problem into smaller tasks is an important step in the design of any parallel algorithm. A natural way to partition the interconnect problem is by using physical partitioning that divides the coupled interconnects into single line subcircuits.

In this section, we review the EMI analysis algorithm based on the lumped segmentation macromodel and WR-TP, suitable for parallel processing [8,35]. This algorithm uses the WR-TP approach to partition the interconnects into single lines. The resulting macromodel will have the equivalent EMI sources given by (2.48)-(2.50) and the WR sources given by (3.21) and (3.37) connected to each lumped segment. These sources can be combined to get the VEEWJ and IEEWJ sources (EEW indicates that 
these are sources obtained by combining equivalent EMI sources and WR sources). The final macromodel with the EEW sources connected to the lumped segments is shown in Figure 5.1.

$$
\begin{aligned}
& V E E W j(Z k, t)=V_{E} E, j\left\{z_{k}, t\right)+e_{k}(Z k, t) \\
& i E E W j(z k, t)=i E E, j(z k, t)+q t c(z k, t)
\end{aligned}
$$

where VEEJ and IEEJ are the equivalent EMI sources for $j^{t h}$ line discussed in Section 2.3.1, e.k and $q \sim k$ are the distributed WR sources discussed in Section 3.2, and $A z$ is the length of each lumped section.

Using this method for a n-line interconnect, we get $n$ subcircuits that can be simulated in parallel. If we consider simulation of each of these subcircuits for a particular line and iteration as one task, then the maximum number of tasks available for parallel execution is $n$. In other words, the maximum concurrency of this approach

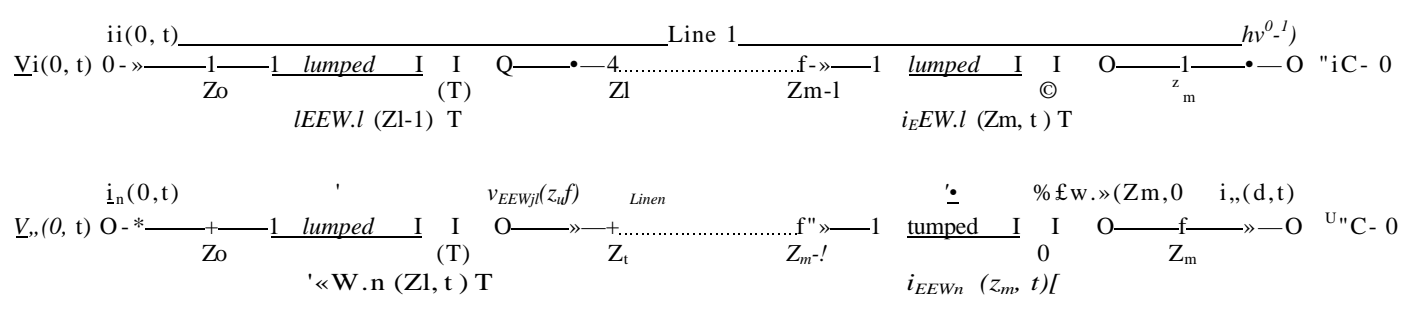

Figure 5.1: EMI macromodel based on WR-TP and lumped segmentation. 
is limited to $n$. This limited concurrency results in poor CPU utilization efficiency because some processors may be starved of tasks. The CPU utilization efficiency 77 for this approach is given by [9]

$$
\boldsymbol{v}=\frac{n}{\boldsymbol{U p}} \quad \begin{array}{ll}
n & \boldsymbol{U}
\end{array}
$$

where $n$ is the number of lines, rip is the number of processors and [.] is the ceiling operator. From (5.3), it is clear that the CPU utilization is less than $100 \%$ when $\mathrm{n}$ is not divisible by $n p$. Next, we discuss the time partitioning approach that can overcome this problem to a great extent.

\subsubsection{Review of Parallel Interconnect Simulation Using Phys- ical and Time Partitioning (PS-PTP)}

The CPU utilization efficiency of the above algorithm can be improved, if there are enough tasks at each stage of execution to avoid having idle processors. More tasks can be created by using a finer decomposition method based on time partitioning [9], used along with the physical partitioning. In time partitioning, the transient simulation of each line is divided into n\# time blocks, and simulation of a particular time block for a particular line and iteration is defined as a task. These tasks can be executed in an order that is determined by the following conditions: 
1. Simulation results from the previous iteration of that particular block should be available, so that the WR sources can be calculated.

2. Simulation results from the previous time block for the current iteration should be available, so that simulation of current time block can be initialized.

The main advantage of using the time partitioning scheme is that the simulation of next iteration of a time block can be started without waiting for the simulation of all other time blocks in the previous iteration to finish.

The CPU utilization efficiency for PS-PTP approach, when $n>n p$ is given by [9]

$$
V=\frac{\text { nnpnj }[\text { [nnpnj }}{n \boldsymbol{p}} \quad \boldsymbol{n p}
$$

where $n p$ is the number of time blocks and $n j$ is the number of WR iterations. From (5.4) it can be shown that a 100\% CPU utilization efficiency can be acheived if $n p=n p$ is chosen [9]. Note that here it is assumed that the simulation tasks are of equal size. In practical circuits, it may not be always possible to divide the simulation tasks into equal sizes, which can result in some idle time for processors, however, the PS-PTP method will provide better efficiency compared to the PS-PP method in most cases. 


\subsection{Proposed Parallel EMI Analysis Algorithm}

Even though the physical partitioning of interconnects facilitates parallelization of interconnect simulation, it does not achieve a good CPU utilization efficiency, as we had noted earlier. Similar is the case of EMI analysis based on physical partitioning. To achieve near optimum utilization of processors, the proposed algorithm partitions the simulation problem into time blocks.

In the proposed algorithm, we start with RLGC parameters of the coupled transmission line along with the geometry of the interconnect structure and the details of the plane wave incidence. From these parameters, the distributed EMI sources are calculated using the field coupling models discussed in Chapter 2. Next, these coupled lines are partitioned into single line subcircuits using the WR-TP method. These single lines are represented as lumped circuits using appropriate transmission line macromodel. Once the MNA for these circuits are built in the shared memory, the transient simulation can be performed.

It is to be noted that transient simulation is divided into time blocks, and simulation of each time block for a particular line and iteration constitutes a single task. The number of time blocks $n \#$ required is determined by the number of processors available $n p$. As discussed earlier, optimum CPU utilization is obtained when using $U B=n p$.

The tasks are scheduled for execution in an order determined by the conditions 
given in Section 5.1.1. This is done by creating a work pool of all possible tasks ( $\mathrm{n} \times U B \times n j$ tasks) and checking them one by one to identify tasks that are ready for execution. The tasks that are ready for execution are assigned to the available processors.

The execution of an assigned task is started by updating the values of the EEW sources for that particular block using the previous iteration results. In the case of first iteration, an initial guess equal to the value of EMI sources (VEEJ, $\left.\wedge^{\wedge} E B J\right)$ is used. Next, the transient simulation is initialized using the result of previous time block. Then, the subcircuit is simulated for the duration of the time block and the task is removed from the work pool. This process is continued until the work pool has no more tasks left.

These steps described above are summarized in the Pseudocodes 5.1-5.3. Here, the individual tasks are represented by an ordered triplet $(r, l, b)$, where $\mathrm{r}, I$ and $b$ are the iteration, line and time block of the task, respectively. The Pseudocode 5.1 has the main body of the algorithm, where the tasks are created using physical and time partitioning of the interconnect circuit. This main function also schedules the execution of these tasks with help of a workjpool and a task.verify function. The task-verify function(listed in Pseudocode 5.2) ensures that the tasks scheduled for execution are ready for simulation by verifying the required conditions given in Section 5.1.1. Once the tasks are verified and scheduled for execution, the simulation 
of the task is performed by the function performjwr-iteration function listed in

Pseudocode 5.3.

Pseudocode 5.1 Proposed parallel EMI analysis algorithm

1: Use transverse partitioning to divide the coupled interconnect into individual lines.

2: Subdivide the time vector into n\# time blocks.

3: Calculate the EMI sources vector srcsMi-

Define work-pool $=$ NULL $\{$ list of all the simulation tasks $\}$

Define task-results $=$ NULL $\{$ stores the intermediate results between the iterations \}

6: for $\mathrm{r}=1$ to $\mathrm{n} / \mathrm{do}$

7: $\quad$ for $I=1$ to $n$ do

8: $\quad$ for $b=1$ to $T I B$ do

9: $\quad$ append task (r, $I, b)$ to end of work-pool

10: $\quad$ end for

11: end for

12: end for

13: while size of work-pool > 0 do

14: for $p=1$ to $n p$ do

15: $\quad$ if size of work-pool > 0 then

16: $\quad$ current-task $=$ next task from work-pool

17: $\quad$ task-ready $=$ call task-veviiy(current-task,task-results)

18: $\quad$ if task-ready - true then

19: call peviorm.Jwr-iter\&tion(current-task,task-results) on processor $p$

20: remove current-task from work-pool

21: $\quad$ else

22: $\quad$ break out of for loop

23: $\quad$ end if

24: $\quad$ end if

25: end for

26: wait for next task which is ready for execution

27: end while 
Pseudocode 5.2 Function for task flow verification

1: function task-veriiy(current-task,task-results)

2: let $(r, l, b)$ represent the current-task

3: if $b>1$ then

4: if results of $(\mathrm{r}, 1 \ldots \mathrm{n}, b) \wedge$ task-results or $(\mathrm{r}, /, 6-1) \wedge$ task-results then

5: return false

6: end if

7: end if

Pseudocode 5.3 Function for WR iteration

1: function perform.wr_iteration(curren£Jask,task results)

2: let $(r, l, b)$ represent the current-task

3: if $r>1$ then

4: $\quad$ load results for $(\mathrm{r}-1,1 \ldots \mathrm{n}, b)$ from task-results

5: update the EEW sources and the WR sources using (3.21) and (3.37)

6: else

7: use EMI sources $\left(V E E J,{ }^{\wedge} E E J\right)$ as the initial guess for EEW sources

8: end if

9: if $b>1$ then

10: initialize circuit simulation using results of $(r, I, b-1)$ from task-results

11: end if

12: perform transient simulation

13: save results of $(r, l, b)$ in task-results

14: end function 


\subsection{Numerical Examples}

The proposed EMI analysis method was implemented using $\mathrm{C}++$ and OpenMP. The simulation of the partitioned circuits (resulting from WR-TP) are done using an inhouse circuit simulator that provides complete access to intermediate results, so as to reduce the overhead related to calculation of WR sources. The numerical experiments

were executed on a dual Quad-core AMD Opteron 2344HE processor with $2.3 \mathrm{GHz}$ clock, with ccNUMA architecture.

\section{Example 1: 25 Lines Illuminated by a Plane Wave}

The RLGC parameters for this large example are created from the RLGC parameters of the structure in Figure 5.2, by extrapolation. The length of each line is $15 \mathrm{~cm}$. These lines are excited by a plane wave incident at the origin at an angle $9=45^{\circ}, 0=0^{\circ}$ and polarized at $9 E=-90^{\circ}$. The time envelope of the electric field is defined by a Gaussian pulse of amplitude $5 \mathrm{kV} / \mathrm{m}$ and pulse width of Ins. The near and far ends of the lines were terminated using a $60 \mathrm{~J} 7$ resistor and a $l p F$ capacitor, respectively. The Line 1 was also excited using a trapezoidal pulse current source connected at the near end. 


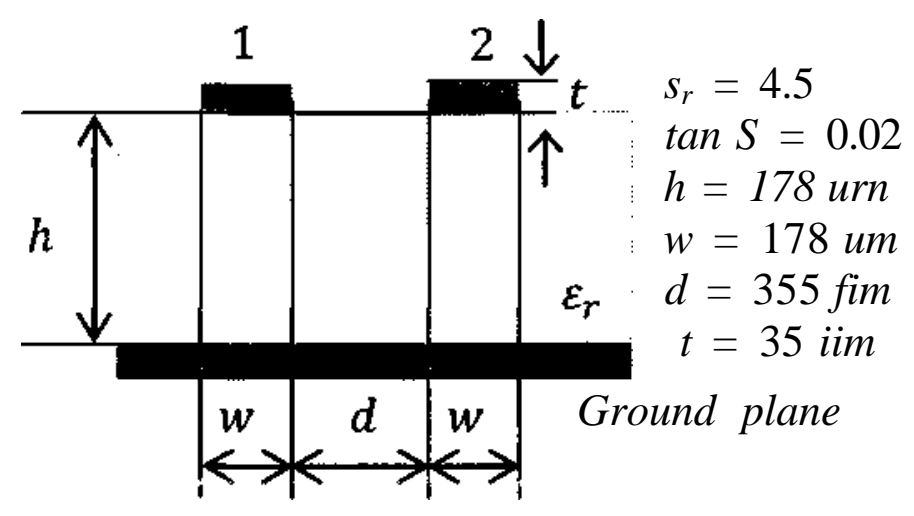

Figure 5.2: 2-line MTL structure.

In this experiment, this MTL was simulated using the proposed EMI analysis method that uses time partitioning, on a multicore machine with $n_{p}$ processors. The MTL was represented using 80 lumped RLC sections and the transient duration was divided into $U Q$ time blocks, satisfying the condition ng $=n p$. The accuracy of the simulation is tested by comparing the results of the proposed parallel EMI analysis method with the conventional method (coupled lumped segmentation) results (shown in Figures 5.3-5.5). These graphs show a good agreement between the proposed and the conventional method results. 


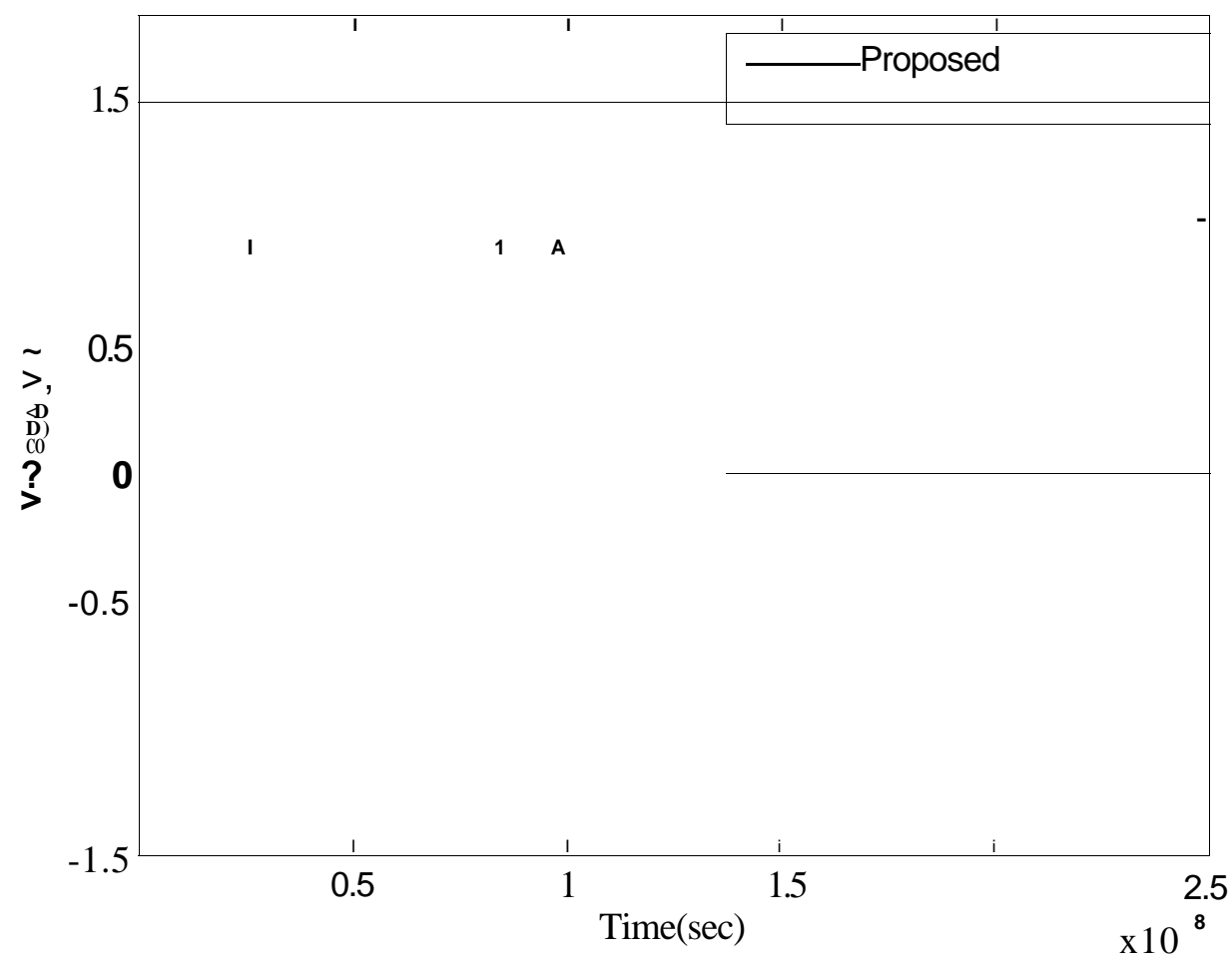

Figure 5.3: Example 1: Comparison of transient responses at the far end of line 1. 


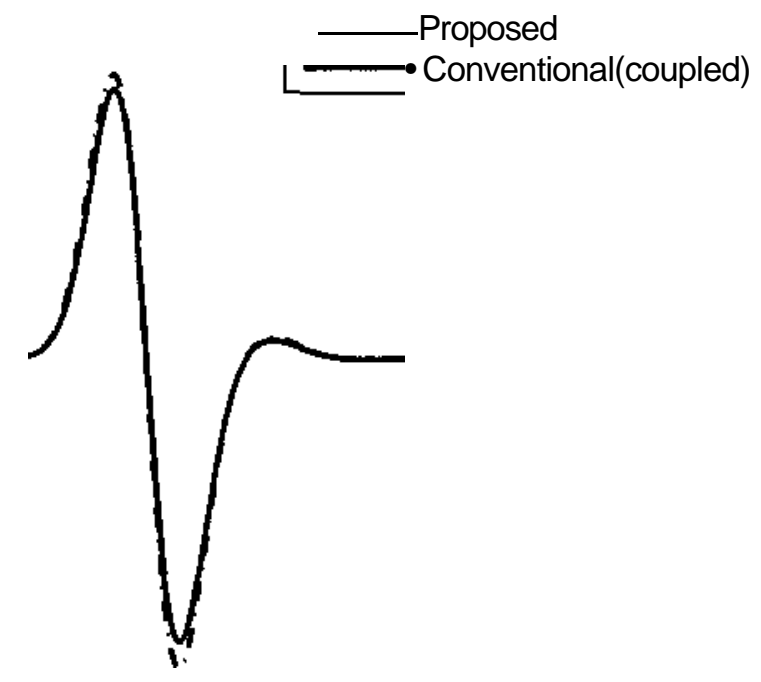

0.5

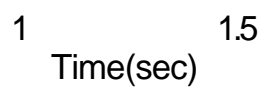

2

2.5 $\mathrm{x} 10^{18}$

Figure 5.4: Example 1: Comparison of transient responses at the far end of line 1 (With EM field excitation alone). 


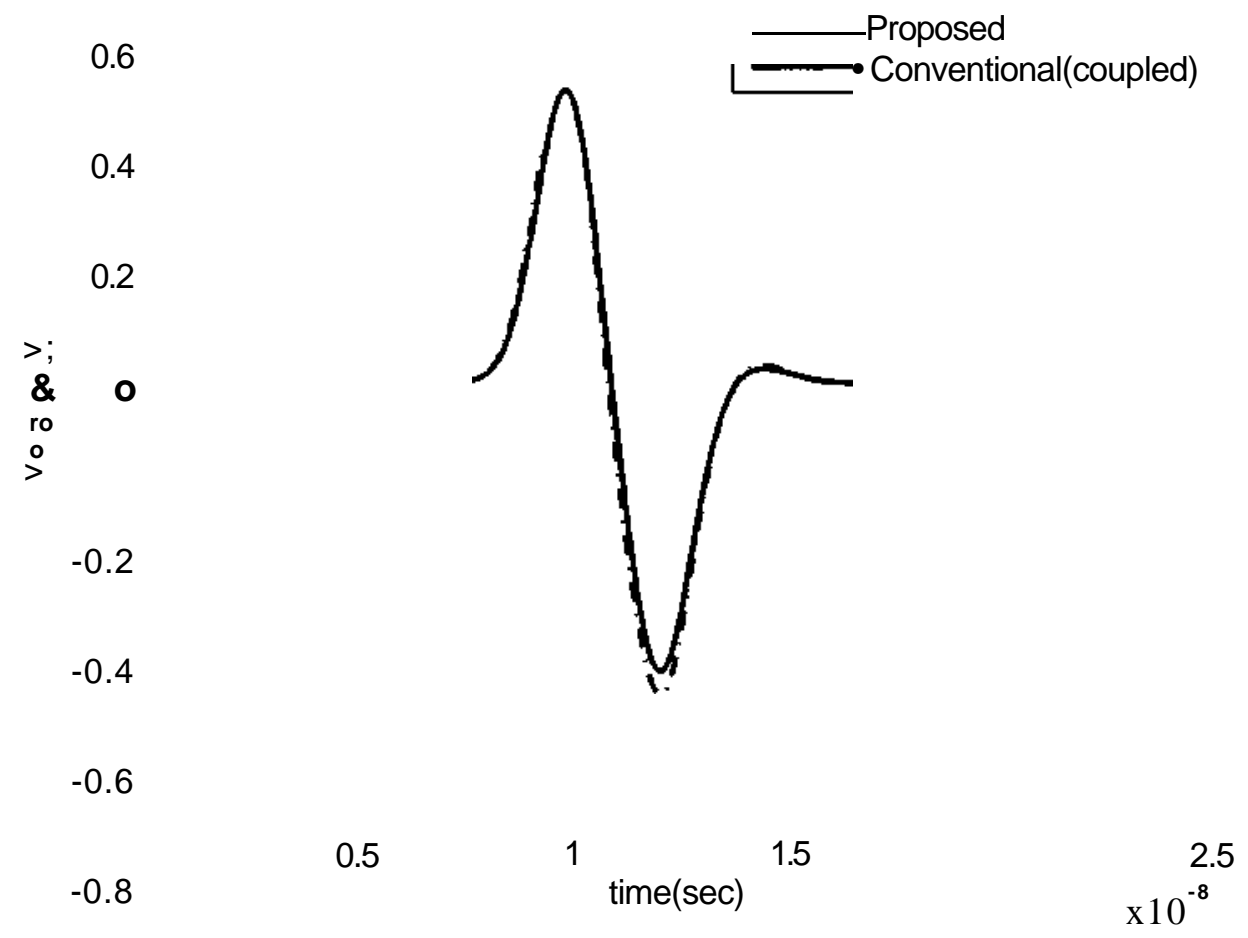

Figure 5.5: Example 1: Comparison of transient responses at the near end of line 25. 


\section{Example 2: Scalability of the proposed parallel algorithm}

\section{w.r.t. number of CPUs}

The scalability of the algorithm with respect to number of processors is tested for the case of a 25-line interconnect structure (same as in Example 1). The run times for simulating this problem using different number of CPUs are listed in the Table 5.1. Here, the speed-up of the parallel algorithm is calculated as

$$
\text { speed'-up }=\frac{\text { simulation time using } n p \text { processors }}{\text { simulation time using one processor }}
$$

The corresponding speed up curve is shown in Figure 5.6. It can be observed that the PS-PTP algorithm attains a good speed-up even when the number of lines simulated is not an integer multiple of the number of processors. 


\begin{tabular}{|c|c|c|}
\hline Number of CPUs & $\begin{array}{c}\text { Proposed method } \\
\text { Simulation time (s) }\end{array}$ & Speed-up \\
\hline 1 & 86.0 & 1.0 \\
\hline 2 & 45.4 & 1.9 \\
\hline 3 & 30.3 & 2.8 \\
\hline 4 & 23.0 & 3.7 \\
\hline 5 & 18.8 & 4.6 \\
\hline 6 & 15.7 & 5.4 \\
\hline 7 & 14.3 & 5.9 \\
\hline 8 & 13.1 & 6.5 \\
\hline
\end{tabular}

Table 5.1: Example 2: Simulation time measurements for proposed parallel EMI analysis method.

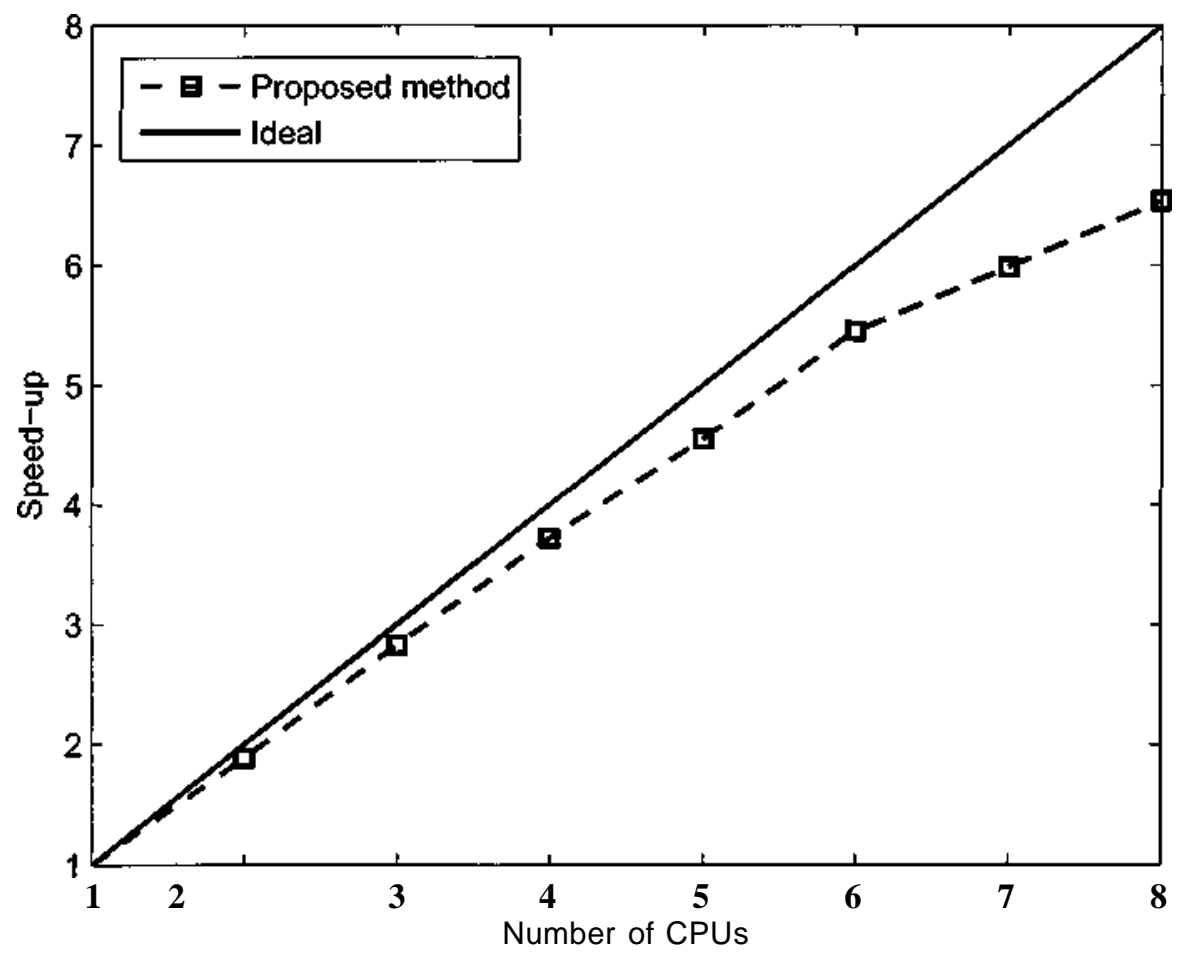

Figure 5.6: Example 2: Speed-up vs number of processors graph for the proposed parallel EMI analysis method. 
Example 3: Scalability of the proposed parallel algorithm w.r.t. number of lines

In this experiment, the proposed algorithm is used to simulate a set of coupled interconnects having different number of lines, and the corresponding simulation times are measured. The experiment is repeated for two cases: with 4 CPUs and with 8 CPUs. The corresponding measurement results are compared with the CPU times of conventional coupled lumped segmentation model in the Figure 5.7. The speed-up measurements are given in Tables 5.2 and 5.3. 


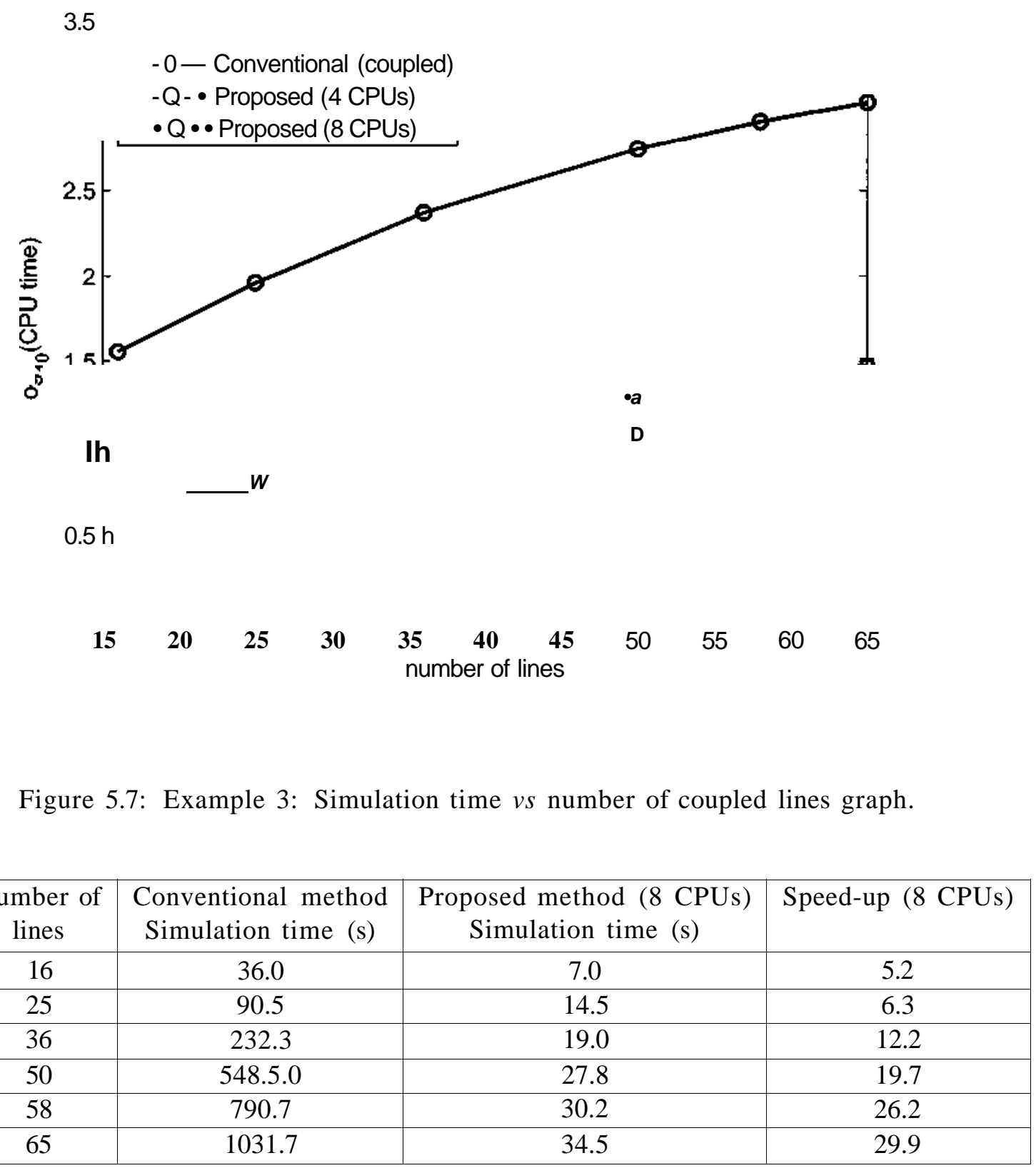

Table 5.2: Example 3: Simulation time for proposed parallel EMI analysis method (8 CPUs) 


\begin{tabular}{|c|c|c|c|}
\hline $\begin{array}{c}\text { Number of } \\
\text { lines }\end{array}$ & $\begin{array}{c}\text { Conventional method } \\
\text { Simulation time(s) }\end{array}$ & $\begin{array}{c}\text { Proposed method (4 CPUs) } \\
\text { Simulation time(s) }\end{array}$ & Speed-up (4 CPUs) \\
\hline 16 & 36.0 & 7.0 & 2.5 \\
\hline 25 & 90.5 & 14.5 & 4.0 \\
\hline 36 & 232.3 & 19.0 & 7.1 \\
\hline 50 & 548.5 .0 & 27.8 & 11.4 \\
\hline 58 & 790.7 & 30.2 & 14.4 \\
\hline 65 & 1031.7 & 34.5 & 16.6 \\
\hline
\end{tabular}

Table 5.3: Example 3: Simulation time for proposed parallel EMI analysis method (4 CPUs)

\subsection{Summary}

A new parallel EMI analysis algorithm for massively coupled interconnects based on WR-TP and time partitioning was presented in this chapter. Numerical experiments with different number of processors showed that the proposed parallel algorithm scales well with number of CPUs and achieves a speed-up close to the ideal speed up achievable. The proposed method gains significant speed-up compared to conventional coupled macromodel, when used to simulate massively coupled interconnects. 


\section{Chapter 6}

\section{Conclusions and Future Work}

This thesis presented novel algorithms for fast EMI analysis of massively coupled interconnects, which are based on existing quasi-TEM field coupling models. These algorithms use waveform relaxation and transverse partitioning to subdivide the interconnect circuit exposed to electromagnetic interference into independent subcircuits, and solve them iteratively, instead of directly solving the large coupled circuit using an LU solver. In addition, the case of long-delay interconnects is handled via DEPACT macromodel that is used to represent the decoupled interconnect subcircuits. The proposed method achieved a linear growth in computational complexity $O(n)$, compared to $O\left(n^{A}\right)$ required by conventional (coupled) methods.

A new parallel algorithm was also presented that extends the existing WR-TP based EMI analysis algorithm via time partitioning, so as to achieve better CPU uti- 
lization on parallel processing platforms. A parallel implementation of this algorithm was built using the OpenMP environment and was tested on a multi-core processor with a ccNUMA architecture. The tests performed using different number of cores showed a good simulation speed-up characteristic w.r.t the number of cores.

\subsection{Future Work}

Several future projects can be undertaken based on the EMI analysis algorithms presented in this thesis. Specifically, these algorithms could be extended to handle a greater variety of interconnects and different types of EMI sources. Advancements can be made mainly on two aspects: (1) the complexity of the field coupling model, (2) the convergence of WR-TP method. Some of these are listed below:

1. Field coupling model: The accuracy of the field coupling model used for EMI analysis could be improved by considering the following effects which are ignored in the current model:

a) Including the effects of multiple interfaces in the medium, when multilayered dielectrics are used in the interconnects [36].

b) Including the "charge and current separation" effects that are considerable when the line separation is comparable to the line width. [1].

c) Including the effects of lossy ground plane [10] to account for the sheet 
resistance of the ground plane.

d) Including the effects of non-plane wave excitations [13].

2. Convergence of WR-TP: The convergence of WR-TP algorithm in the case of tightly coupled lines can be improved by using sliding window partitioning that allows more than one line in each partition [29]. 


\section{References}

[1] F. M. Tesche, M. Ianoz, and T. Karlsson, EMC analysis methods and computational models. $\quad$ New York: John Wiley Sons, 1997.

[2] C. R. Paul, Analysis of multiconductor transmission lines. New York: John Wiley Sons, 1994.

[3] I. Maio, F. G. Canavero, and B. Dilecce, "Analysis of crosstalk and field coupling to lossy MTLs in a spice environment," IEEE Transactions on Electromagnetic Compatibility, vol. 38, no. 3, pp. 221-229, Aug 1996.

[4] I. Erdin, A. Dounavis, R. Achar, and M. Nakhla, "A spice model for incident field coupling to lossy multiconductor transmission lines," IEEE Transactions on Electromagnetic Compatibility, vol. 43, no. 4, pp. 485 494, Nov 2001.

[5] G. S. Shinh, N. M. Nakhla, R. Achar, M. S. Nakhla, A. Dounavis, and I. Erdin, "Fast transient analysis of incident field coupling to multiconduc- 
tor transmission lines," IEEE Transactions on Electromagnetic Compatibility, vol. 48, no. 1, pp. 57-73, Feb 2006.

[6] N. M. Nakhla, A. E. Ruehli, M. S. Nakhla, and R. Achar, "Simulation of coupled interconnects using waveform relaxation and transverse partitioning," IEEE Transactions on Advanced Packaging, vol. 29, no. 1, pp. 78-87, Feb 2006.

[7] N. Nakhla, A. Dounavis, R. Achar, and M. Nakhla, "DEPACT: delay extraction-based passive compact transmission-line macromodeling algorithm," IEEE Transactions on Advanced Packaging, vol. 28, no. 1, pp. 13 -23, Feb 2005.

[8] A. R. Sridhar, N. M. Nakhla, R. Achar, M. S. Nakhla, and A. E. Ruehli, "Fast EMI analysis via transverse partitioning and waveform relaxation," IEEE Transactions on Electromagnetic Compatibility, vol. 51, no. 2, pp. 358-371, May 2009.

[9] D. Paul, N. M. Nakhla, R. Achar, and M. S. Nakhla, "Parallel simulation of massively coupled interconnect networks," IEEE Transactions on Advanced Packaging, vol. 33, no. 1, pp. 115-127, Feb 2010.

[10] R. Perez, Handbook of Electromagnetic Compatibility. San Diego: Academic Press, 1995. 
[11] R. Achar and M. S. Nakhla, "Simulation of high-speed interconnects," Proceedings of the IEEE, vol. 89, no. 5, pp. 693-728, May 2001.

[12] C. Taylor, R. Satterwhite, and C. H. Jr., "The response of a terminated two-wire transmission line excited by a nonuniform electromagnetic field," IEEE Transactions on Advanced Packaging, vol. 13, no. 6, pp. 987-989, Nov 1965.

[13] A. K. Agrawal, H. J. Price, and S. H. Gurbaxani, "Transient response of multiconductor transmission lines excited by a nonuniform electromagnetic field," IEEE Transactions on Electromagnetic Compatibility, vol. EMC-22, no. 2, pp. 119-129, Feb 1980.

[14] A. A. Smith, "A more convenient form of the equations for the response of a transmission line excited by nonuniform fields," IEEE Transactions on Electromagnetic Compatibility, vol. EMC-15, no. 3, pp. 151-152, Aug 1973.

[15] C. R. Paul, "Frequency response of multiconductor transmission lines illuminated by an electromagnetic field," IEEE Transactions on Electromagnetic Compatibility, vol. EMC-18, no. 4, pp. 183-190, Nov 1976.

[16] Y. Bayram and J. Volakis, "A generalized mom-spice iterative technique for field coupling to multiconductor transmission lines in presence of complex 
structures," IEEE Transactions on Electromagnetic Compatibility, vol. 47, no. 2, pp. 234 - 246, May 2005.

[17] A. Karwowski, "Broadband analysis of electromagnetic field coupling to transmission lines," in 15th International Conference on Microwave Techniques (COMITE), Apr 2010, pp. $183-184$.

[18] HSPICE signal integrity user guide, Synopsis, Version C-2009.03, March 2009.

[19] G. S. Shinh, R. Achar, N. M. Nakhla, M. S. Nakhla, and I. Erdin, "Simplified macromodel of mtls with incident fields SiMMIF," IEEE Transactions on Electromagnetic Compatibility, vol. 50, no. 2, pp. 375-389, May 2008.

[20] T. Dhaene and D. de Zutter, "Selection of lumped element models for coupled lossy transmission lines," IEEE Transactions on Computer-Aided Design of Integrated Circuits and Systems, vol. 11, no. 7, pp. $805-815$, Jul 1992.

[21] J. Branin, F.H., "Transient analysis of lossless transmission lines," Proceedings of the IEEE, vol. 55, no. 11, pp. 2012 - 2013, Nov. 1967.

[22] A. Dounavis, R. Achar, and M. Nakhla, "A general class of passive macromodels for lossy multiconductor transmission lines," IEEE Transactions 
on Microwave Theory and Techniques, vol. 49, no. 10, pp. $1686-1696$, Oct 2001.

[23] E. Gad, "Circuit-based analysis of electromagnetic field coupling with nonuniform transmission lines," IEEE Transactions on Electromagnetic Compatibility, vol. 50, no. 1, pp. 149 -165, Feb 2008.

[24] V. Vahrenholt, H. Bruns, R. Krzikalla, and H. Singer, "Fast analysis of electromagnetic field coupling into pcbs based on the peec method," in International Symposium on Electromagnetic Compatibility - EMC Europe,, Sep 2008, pp. $1-6$.

[25] G. S. Shinh, "Closed-form macromodels for analysis of high-speed interconnects in the presence of electromagnetic fields," Master's thesis, Carleton University, Ottawa, ON, 2005.

[26] R. Bhatia, Matrix analysis. New York: Springer, 1996.

[27] E. Lelarasmee, A. E. Ruehli, and A. L. Sangiovanni-Vincentelli, "The waveform relaxation method for time-domain analysis of large scale integrated circuits," IEEE Transactions on Computer-Aided Design of Integrated Circuits and Systems, vol. 1, no. 3, pp. 131-145, Jul 1982. 
[28] E. Lelarasmee, "The waveform relaxation method for time domain analysis of large scale integrated circuits," Ph.D. dissertation, University of California, Berkeley, 1982.

[29] N. M. Nakhla, "Waveform relaxation and transverse partitioning algorithms for simulation of massively coupled interconnects," Ph.D. dissertation, Carleton University, Ottawa, ON, 2008.

[30] N. Nakhla, M. Nakhla, and R. Achar, "Simplified delay extraction-based passive transmission line macromodeling algorithm," IEEE Transactions on Advanced Packaging, vol. 33, no. 2, pp. 498-509, May 2010.

[31] A. Narayanan, R. Achar, N. Nakhla, and M. Nakhla, "Fast EMI analysis of massively coupled interconnects with long delay," in Proc. IEEE AsiaPacific Symposium on Electromagnetic Compatibility, 2010, APEMC 2010, May 2010, pp. 618 -621 (Best Student Paper Award).

[32] I. Erdin, "Signal integrity analysis of high-speed circuits with embedded electromagnetic modules," Ph.D. dissertation, Carleton University, Ottawa, ON, 2001.

[33] "The OpenMP website," 2010. [Online]. Available: http://openmp.org/ 
[34] Mentor ELDO users manual, Mentor Graphics Corporation, Version 2008.2a.

[35] R. Achar, A. Sridhar, N. Nakhla, and M. Nakhla, "Parallel EMI analysis of large coupled interconnects via transverse partitioning and waveform relaxation," in Proc. IEEE Asia-Pacific Symposium on Electromagnetic Compatibility and 19th International Zurich Symposium on Electromagnetic Compatibility, 2008, APEMC 2008, May 2008, pp. 490-493.

[36] I. Erdin, R. Khazaka, and M. S. Nakhla, "Simulation of high-speed interconnects in a multilayered medium in the presence of incident field," IEEE Transactions on Microwave Theory and Techniques, vol. 46, no. 12, pp. 2251-2257, Dec 1998. 